\title{
Quadrupedal Locomotion using Hierarchical Operational Space Control
}

\section{Journal Article}

\section{Author(s):}

Hutter, Marco (1); Sommer, Hannes; Gehring, Christian; Hoepflinger, Mark A.; Bloesch, Michael; Siegwart, Roland

Publication date:

2014

Permanent link:

https://doi.org/10.3929/ethz-a-010184871

Rights / license:

In Copyright - Non-Commercial Use Permitted

Originally published in:

The International Journal of Robotics Research 33(8), https://doi.org/10.1177/0278364913519834 


\title{
Quadrupedal Locomotion using Hierarchical Operational Space Control
}

\author{
Marco Hutter, Hannes Sommer, Christian Gehring, Mark Hoepflinger, Michael Bloesch, Roland Siegwart \\ Autonomous Systems Lab, ETH Zurich, Switzerland, mahutter@ethz.ch *
}

December 17, 2013

\begin{abstract}
This paper presents the application of operational space control based on hierarchical task optimization for quadrupedal locomotion. We show how the behavior of a complex robotic machine can be described by a simple set of least squares problems with different priorities for motion, torque, and force optimization. Using projected dynamics of floating base systems with multiple contact points, the optimization dimensionality can be reduced or decoupled such that the formulation is purely based on the inversion of kinematic system properties. The present controller is extensively tested in various experiments using the fully torque controllable quadrupedal robot StarlETH. The load distribution is optimized for static walking gaits to improve contact stability and/or actuator efficiency under various terrain conditions. This is augmented with simultaneous joint position and torque limitations as well as with an interpolation method to ensure smooth contact transitions. The same control structure is further used to stabilize dynamic trotting gaits under significant external disturbances such as uneven ground or pushes. To the best of our knowledge, this work is the first documentation of static and dynamic locomotion with pure task-space inverse dynamics (no joint position feedback) control.
\end{abstract}

\section{Introduction}

Research in the field of legged robotic systems has recently made significant progress both in the field of hardware design as well as in control development. Traditional (industrial) robotic devices with precise, high-gain position control get more and more replaced by torque controllable robots that can softly (and hence safely) interact with their environment (e.g. Hirzinger et al. 2001). This fundamental change opens wide opportunities to bring novel control concepts from simulations into real world applications. Instead of slow, static walking based on kinematic motion planning and execution (Gonzales de Santos et al., 2006), these new principles have the potential to enable agile, highly dynamic, and versatile maneuvers. Torque controllability allows to create sophisticated behaviors by simultaneously emulating and optimizing desired task-space dynamics, joint torques, or contact forces.

A particularly interesting and widely used approach is inverse dynamics. Given a desired motion, the multi-body system dynamics of the robot is inverted to generate required joint torques. While this works straight forward for fixed base manipulators, it is only recently applied to floating base systems such as legged robots, i.e. to systems that are not rigidly tied to the environment and that are hence underactuated. Most floating base inverse dynamics methods rely on pseudo-inversion. First, the system dynamics are mapped onto a support-consistent manifold using methods such as the dynamically consistent support null-space (Sentis, 2007), linear projection (Aghili, 2005), orthogonal projection (Mistry et al. 2010), or others. Subsequently, this reduced dimensional description is inverted. As it was shown by Righetti et al. (2010, 2011b, 2013), the underlying pseudo-inversion can be weighted to additionally minimize a linear combination of quadratic costs in contact forces and joint torques.

To establish the desired motion respectively behavior of complex systems with a large number of degrees of freedom in an intuitive way, the desired dynamics can be prescribed only at particularly interesting points instead for the complete system (Samson et al., 1991). To give some examples, this can be controlling the center of gravity (CoG) to ensure stability, performing stepping or grasping tasks with hands or feet, keeping a certain optimal posture, or applying a desired contact force. This is called task- or operational space control (OSC). Using different control gains and priorities, it can be ensured that important or high-precision tasks are accurately executed while other, less important tasks are only fulfilled as well as possible without impairing the ones with higher priority. Such a differentiation is not a purely robotic construct: Various behavioral and neuro-scientific studies (e.g. Saltzman and Kelso, 1987, Schaal and Schweighofer, 2005, Scholz and Schoner, 1999) have indicated that humans show a very similar task decomposition with high-gain control for e.g., a reaching task with the hand and compliant null space posture control. In robotics, such prioritized control formulations were first proposed by Hanafusa et al.(1981) and Nakamura et al.(1987) in the context of inverse kinematics control and later picked up by Siciliano and Slotine (1991) who generalized it for an arbitrary number of tasks. Along the lines of the

\footnotetext{
*This work was supported in part by the the Swiss National Science Foundation through the National Centre of Competence in Research Robotics as well as by the Hans Eggenberger Foundation.
} 


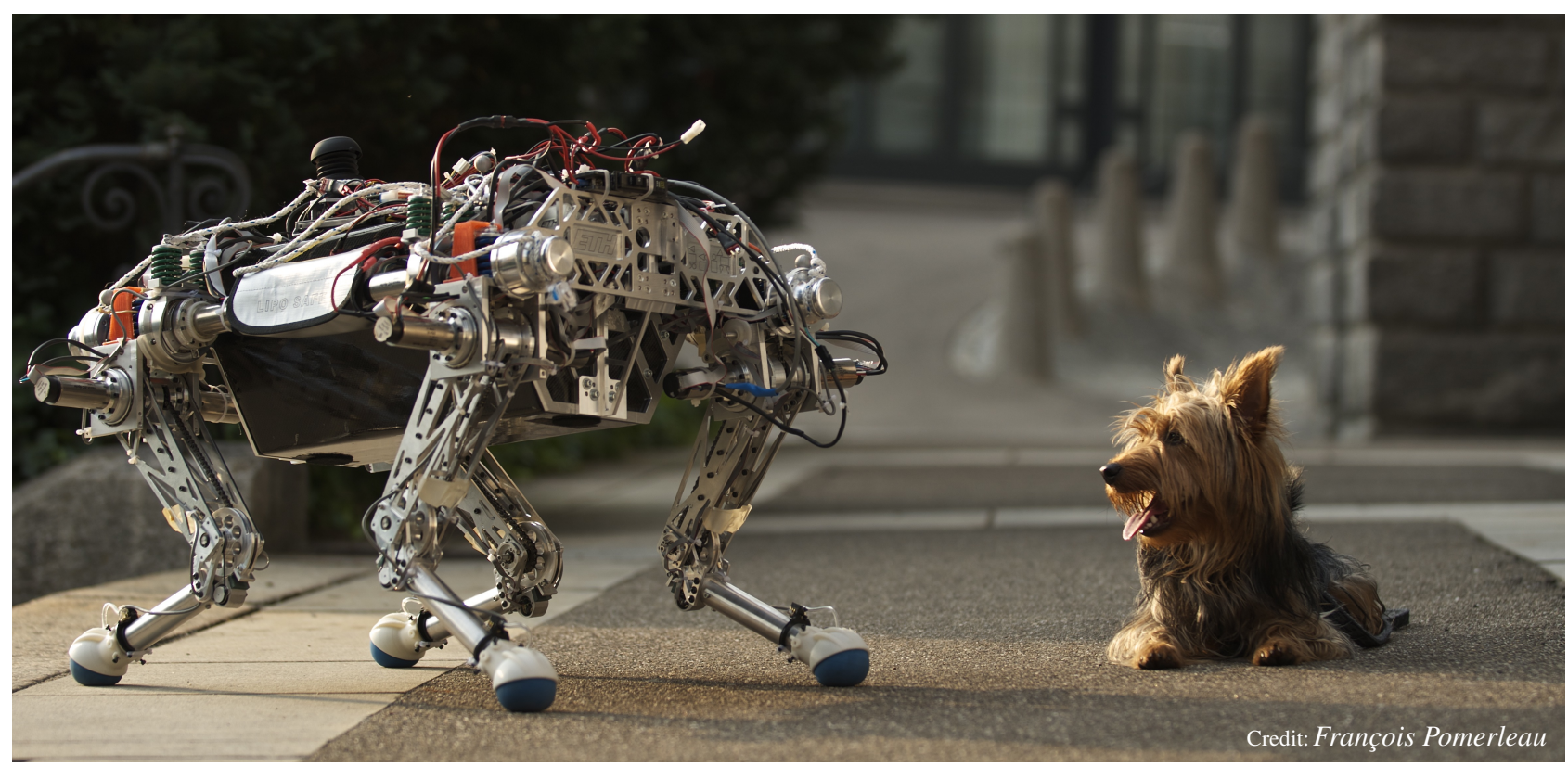

Figure 1: StarlETH is a medium dog-sized quadrupedal robot driven by precisely torque controllable series elastic actuators (Hutter et al., 2012a).

seminal work on Operational Space Control (OSC) by Khatib (1987), his group extended the task prioritization ideas to hierarchical OSC for floating-base systems. They set up a software framework to solve task-space inverse dynamics with various priorities for both motion as well as force/torque tasks (Sentis and Khatib, 2010). Philippsen et al. (2011) recently released this powerful software package for public. Similar methods for motion tasks were also published by de Lasa and Hertzmann (2009) based on the formulation of a minimization problem. All these hierarchical control frameworks guarantee by iterative null-space projection that a task never impairs a higher prioritized task. Other methodologies (e.g. Escande et al. 2010) solve the hierarchy of tasks with numerical solvers (Björck, 1996) for constrained quadratic programs (QP) to fast and robustly handle the redundancy as it can occur in complex robotic systems. Similar to the iterative null-space projection, dedicated QP formulations (Mansard, 2012) are applied for inverse dynamics methods (e.g. Ramos et al., 2012, Saab et al., 2011a b, 2013, ?). Moreover, inequality tasks (Mansard et al., 2009) as required to describe position, torque, or obstacle limitations, can be very naturally integrated using slack variables. This was shown by Kanoun et al. (2011) for kinematic problems and Saab et al. (2013) later suggested to augment this method for inverse dynamics control.

All existing whole-body control techniques share the problem that, due to the lack of appropriate robotic devices, they are largely limited to run in simulations, on very limited torque controlled wheeled platforms (?), or require the co-generated generalized joint accelerations as actuator commands (Saab et al. 2013). Moreover, existing experimental studies that investigated inverse dynamics methods in real hardware experiments reveal some difficulties. This is for example documented by Nakanishi et al. (2008), who compared different (task-space) control techniques in a thorough experimental study on a single fixed base hydraulic Sarcos Master Arm robot. One of the findings was that OSC exhibits a significant dependence on a highly accurate model identification of the robot. Methods as (Khatib, 1987) work perfectly in simulations but largely suffer from modeling errors in hardware experiments. The inversion of the rigid body dynamics inertia matrix is identified as one of the critical components responsible for this degradation since it creates many sources of errors in the motor commands due to its appearance in the pseudo-inverse. Although the achieved performance certainly depends on the implementation, the demonstration of the capability of such methods in complex legged systems is still missing.

The contribution of this paper is twofold. First, based on different existing whole-body respectively inverse dynamics control techniques, we outline a clean and simple framework for hierarchical OSC. All tasks for motion, torque, and force optimization are expressed as least squares problems and can hence be solved by iterative null-space projection or standard numerical QP solvers. We illustrate how the optimization dimensionality can be reduced by tricks of projected equations of motion, how these formulations relate to classic kinematic task prioritization paired with inverse dynamics control, and give an overview how they are employed and how they compare. Second, we apply and extensively test the proposed control structure on a fully torque controllable quadrupedal robot StarlETH that we developed for dynamic all-terrain locomotion (Hutter et al. 2012a. Figure 1). This machine is driven by compliant and precisely torque controllable series elastic actuators (SEA) in all twelve joints, which makes it perfectly suited for the presented control techniques. We can particularly exploit the multi-contact situation respectively internal contact directions, e.g. to increase energetic efficiency, to lower the risk of slippage, to ensure smooth contact transitions, or to walk on uneven terrain. We employ static and dynamic gaits and show that the proposed control method can robustly stabilize the machine under significant external 


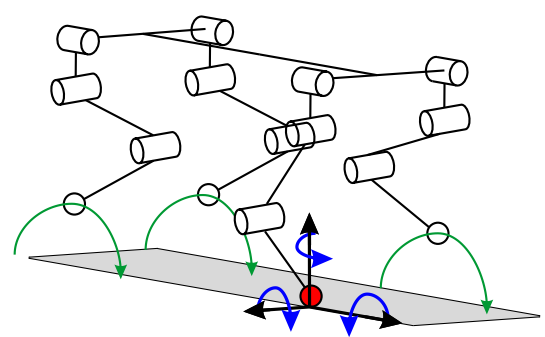

(a) truly-underactuated

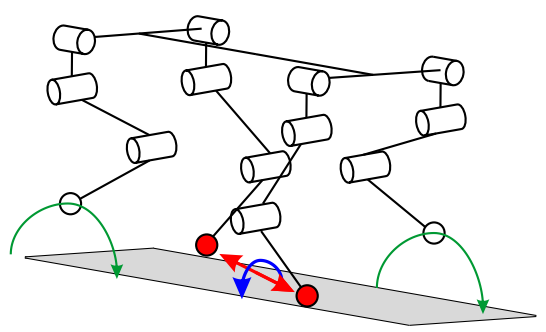

(b) truly-underactuated and overconstrained

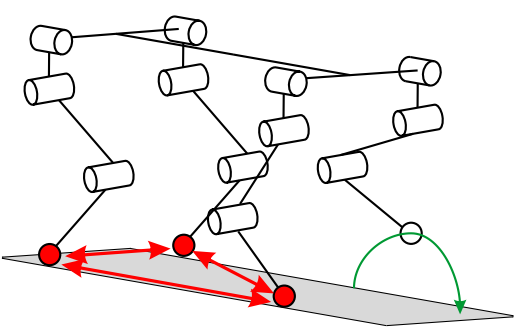

(c) overconstrained

Figure 2: A quadruped robot with point feet is truly-underactuated ((a); $\left.n_{c}=0, n_{u}=3\right)$ in single leg support, trulyunderactuated and overconstrained ((b); $\left.n_{c}=1, n_{u}=1\right)$ in double support, and overconstrained $\left((\mathrm{c}) ; n_{c}=3, n_{u}=0\right)$ during static walking with three simultaneous points of contact.

disturbances. In all experiments, we rely on pure task-space control (no joint position stabilization) and on full onboard sensing respectively state estimation - a result that constitutes that (largely investigated) whole-body control approaches are feasible to regulate future robots. For illustration, all experiments are documented in Extension 1.

\section{Inverse Dynamics of Floating Base Systems}

The system dynamics in terms of equations of motion (EoM) of a floating-base system are described in the form

$$
\mathbf{M} \ddot{\mathbf{q}}+\mathbf{h}+\mathbf{J}_{s}^{T} \mathbf{F}_{s}=\mathbf{S}^{T} \boldsymbol{\tau}
$$

with the mass matrix $\mathbf{M}(\mathbf{q})$, the force vector $\mathbf{h}(\mathbf{q}, \dot{\mathbf{q}})$ as the sum of Coriolis, centrifugal, and gravitational forces, the ground contact forces $\mathbf{F}_{s}$, the corresponding Jacobian $\mathbf{J}_{s}(\mathbf{q})$, and the actuator torque $\boldsymbol{\tau}$. Everything is expressed as a function of the $n$ generalized coordinates $\mathbf{q}=\left(\begin{array}{lll}\mathbf{q}_{b}^{T} & \mathbf{q}_{r}^{T}\end{array}\right)^{T}$, whereby the selection matrix $\mathbf{S}=\left[\begin{array}{ll}\mathbf{0}_{n_{r} \times n_{b}} & \mathbf{I}_{n_{r}}\end{array}\right]$ separates the $n_{r}=n-n_{b}$ actuated joint coordinates $\mathbf{q}_{r}$ from the $n_{b}=6$ unactuated floating-base coordinates $\mathbf{q}_{b}$. Using a linear operator $\mathbf{P}_{F}(\mathbf{q})$ with the properties

$$
\mathbf{P}_{F} \mathbf{J}_{s}^{T}=\mathbf{0}, \quad \operatorname{rank}\left(\mathbf{P}_{F}\right)=\operatorname{dim}\left(\operatorname{ker}\left(\mathbf{J}_{s}\right)\right)=: n_{m},
$$

the contact forces $\mathbf{F}_{s}$ can be eliminated such that the reduced dynamics is written as

$$
\mathbf{P}_{F}(\mathbf{M} \ddot{\mathbf{q}}+\mathbf{h})=\mathbf{P}_{F} \mathbf{S}^{T} \boldsymbol{\tau}
$$

A possible and frequently used choice for the linear operator $\mathbf{P}_{F}$ defined in 2 is an orthogonal projection into the nullspace of $\mathbf{J}_{s}$ (see for example Aghili 2005). For this reason, and similar to most literature in the field, we generally refer to it as projection matrix. As it was shown by Righetti et al. (2011a), different methods like OSC (Sentis and Khatib, 2005), QR decomposition (Mistry et al., 2010), or direct null-space calculation (Aghili, 2005) can be brought into this form. In fact, the choice of projector does not change the resulting control command $\tau$ nor the cost function that is optimized for.

Given desired generalized accelerations $\ddot{\mathbf{q}}$ (motion planning), the joint torque is calculated by pseudo-inversion

$$
\boldsymbol{\tau}=\left(\mathbf{P}_{F} \mathbf{S}^{T}\right)^{+} \mathbf{P}_{F}(\mathbf{M} \ddot{\mathbf{q}}+\mathbf{h})+\mathbf{N}_{P S} \boldsymbol{\tau}_{0},
$$

with the null-space projector $\mathbf{N}_{P S}=\mathcal{N}\left(\mathbf{P}_{F} \mathbf{S}^{T}\right)$ and the arbitrary internal torque vector $\boldsymbol{\tau}_{0}$. Analyzing the matrix $\mathbf{P}_{F} \mathbf{S}^{T}$ respectively the support Jacobian $\mathbf{J}_{s}=\left[\begin{array}{ll}\mathbf{J}_{s_{b}} & \mathbf{J}_{s_{r}}\end{array}\right]$ implies two important differentiations:

Definition 1 (truly-underactuated). A system is called truly-underactuated if there are not enough ground contact constraints to independently control the $n_{b}$ unactuated base coordinates $\mathbf{q}_{b} . n_{u}:=n_{m}-\operatorname{rank}\left(\mathbf{P}_{F} \mathbf{S}^{T}\right)=n_{b}-\operatorname{rank}\left(\mathbf{J}_{s_{b}}\right)>$ 0 coordinates remain uncontrollable.

This entails that the pseudo-inversion may not fulfill the desired dynamics but only minimizes $\| \mathbf{P}_{F}(\mathbf{M} \ddot{\mathbf{q}}+\mathbf{h})-$ $\mathbf{P}_{F} \mathbf{S}^{T} \boldsymbol{\tau} \|_{2}^{2}$. In this case, special attention has to be paid when choosing the desired accelerations $\ddot{\mathbf{q}}$ such that they are dynamically consistent.

Definition 2 (overconstrained). A system is called overconstrained if there exist $n_{c}:=n_{r}-\operatorname{rank}\left(\mathbf{P}_{F} \mathbf{S}^{T}\right)=\operatorname{rank}\left(\mathbf{J}_{s}\right)-$ $\operatorname{rank}\left(\mathbf{J}_{s_{b}}\right)>0$ internal force directions respectively a null-space projector with $\operatorname{rank}\left(\mathbf{N}_{P S}\right)=n_{c}>0$.

This means that the torque vector $\tau$ can be changed by an arbitrary internal torque vector $\tau_{0}$ without impairing the desired acceleration $\ddot{\mathbf{q}}$. Instead, these internal torques $\tau_{0}$ can be used to optimize the load distribution. 
A quadrupedal robot is the appropriate platform to highlight these different contact properties. In contrast to most bipedal systems that have extended foot elements which constrain position and orientation of the foot segment, we employ on StarlETH only point feet. Each contact point implies three independent contact constraints that allow controlling some of the six unactuated base coordinates.

The most simple case with a single leg in contact that constrains only three degrees of freedom corresponds to a trulyunderactuated system with $n_{u}=3$ uncontrollable directions (Figure 2(a). As the robot cannot apply torque around the point contact, the three rotational directions remain uncontrolled. This formulation captures also singular cases such as three contact points in a line, which result in a rank deficiency of $\mathbf{J}_{s_{b}}$.

An overconstrained situation can be realized with three (or more) contact points that are not aligned in a singular configuration (e.g. on a single line). As depicted in Figure 2(c), the $n_{c}=3$ internal directions result from 9 independent contact constraints with only 6 unactuated base coordinates. Thereby, the robot can produce internal forces between the contact points which has no influence on the robot acceleration.

A quadrupedal robot can also be truly-underactuated and overconstrained at the same time (Figure 2(b)): Two simultaneous contact points allow to generate a single internal force between them. At the same time, the system is not able to apply a momentum around the line of support.

\section{Hierarchical Least Squares Optimization}

To prepare for prioritized OSC, we outline in this section the concept of hierarchical least squares optimization of a set of $n_{T}$ systems of linear equations

$$
\mathbf{A}_{i} \mathbf{x}=\mathbf{b}_{i}
$$

with the optimization variable $\mathbf{x}$. Problems of the same priority $i \geq 1$, with $i=1$ being the highest priority, are stacked in matrix $\mathbf{A}_{i}$ and vector $\mathbf{b}_{i}$. As it will be shown later, the EoM as well as motion, joint torque, and contact force tasks can be brought into this linear form, whereby the optimization variable is a stacked vector of generalized acceleration, joint torques, and contact forces. In the proposed hierarchical framework, the goal is to solve each task as good as possible in a least squares sense

$$
\min _{\mathbf{x}}\left\|\mathbf{A}_{i} \mathbf{x}-\mathbf{b}_{i}\right\|_{2}^{2},
$$

without influencing tasks of higher priority, which can be written as

$$
\begin{array}{ll}
\min _{\mathbf{x}} & \left\|\mathbf{A}_{i} \mathbf{x}-\mathbf{b}_{i}\right\|_{2}^{2} \\
\text { s.t. } & {\left[\begin{array}{c}
\mathbf{A}_{1} \\
\vdots \\
\mathbf{A}_{i-1}
\end{array}\right]} \\
& \underbrace{\left(\begin{array}{c}
\mathbf{b}_{1} \\
\vdots \\
\mathbf{b}_{i-1}
\end{array}\right)}_{\hat{\mathbf{A}}_{i-1}}=\underbrace{}_{\hat{\mathbf{b}}_{i-1}} \mathbf{c} .
\end{array}
$$

Note: As long as $\hat{\mathbf{A}}_{i-1}$ has full column rank, the cost $\mathbf{c}$ is $\mathbf{0}$.

This sequence of least squares problems can be solved using a standard quadratic program (QP) solver (Björck, 1996), or analytically and in a very compact way by iterative null-space projection as outlined in the following section.

\subsection{Iterative Null-Space Projection}

As already similarly illustrated by Siciliano and Slotine (1991), the requirement that a task is not allowed to influence any task with higher priority can be formulated by defining $\mathbf{x}$ as a sum of task specific $\mathbf{x}_{i}$ pre-multiplied with the null-space projection matrix $\mathbf{N}_{i}$ of higher prioritized tasks:

$$
\mathbf{x}=\sum_{k=1}^{n_{T}} \mathbf{N}_{k} \mathbf{x}_{k}
$$

The null-space projector $\mathbf{N}_{i}$ is defined as $\mathbf{N}_{i}=\mathcal{N}\left(\left[\begin{array}{lll}\mathbf{A}_{1}^{T} & \ldots & \mathbf{A}_{i-1}^{T}\end{array}\right]^{T}\right)$ with $\mathbf{N}_{1}=\mathbf{I}$ and the sufficient property

$$
\mathbf{A}_{i} \mathbf{N}_{j}=\mathbf{0} \quad \forall i<j .
$$

It can be computed using the pseudo-inverse as outlined in (e.g. Sentis, 2007; Siciliano and Slotine, 1991), which is the analytically correct way of writing an orthogonal null-space projector, or a singular value decomposition (SVD) (Hutter. 
2013, App. A.4), which shows better numerical stability if $\mathbf{A}_{i}$ is close to singular. Using property (9), the prioritized minimization problem (6) can be solved for each task individually by inserting (8) and solving for $\mathbf{x}_{i}$ :

$$
\begin{aligned}
\mathbf{A}_{i} \mathbf{x}-\mathbf{b}_{i} & =\mathbf{A}_{i} \sum_{k=1}^{n_{T}} \mathbf{N}_{k} \mathbf{x}_{k}-\mathbf{b}_{i} \\
\mathbf{x}_{i} & =\left(\mathbf{A}_{i} \mathbf{N}_{i}\right)^{+}\left(\mathbf{b}_{i}-\mathbf{A}_{i} \sum_{k=1}^{i-1} \mathbf{N}_{k} \mathbf{x}_{k}\right)
\end{aligned}
$$

It can be analytically proofed that (i) tasks with higher priority are not influenced by tasks with lower priority and (ii) that the solution is globally optimal (Hutter, 2013, App. A.1). Furthermore, as long as the rows of $\mathbf{A}_{i} \mathbf{N}_{i}$ are linearly independent, the task is exactly fulfilled $\left(\mathbf{A}_{i} \mathbf{x}=\mathbf{b}_{i}\right)$. To ensure numerical robustness also in case the $\mathbf{A}_{i} \mathbf{N}_{i}$ being rank deficient, we use the pseudo-inversion using SVD as described in (?).

\subsection{Inequality Constraints}

To describe the behavior of complex legged robots, it is often required to integrate inequality constraints

$$
\mathbf{A}_{i} \mathbf{x} \leq \mathbf{b}_{i}
$$

in the proposed control framework, e.g. to handle joint angle limitation or motor torque saturation on task level (Mansard et al. 2009, ?). As outlined by Kanoun et al. (2011), such inequality tasks can be naturally integrated with numerical QP solvers using slack variables. An alternative based on the analytical solution is to activate the corresponding inequality task as equality tasks as soon as the inequality constraint is violated. By assigning a higher priority than to all other tasks, we can ensure that (in case there is a feasible solution to the problem) the actual solution fulfills the inequality constraint. While this compact iterative analytical approach works well for our application with a low number of inequality constraints, it is inappropriate to handle multiple independent inequality tasks. The iteration through all possible combinations of active and inactive constraints quickly becomes computationally intensive.

\section{Behavior as Least Squares Problems and Inequality Constraints}

The behavior of a robotic system and in particular legged robots can be described as simultaneous execution of multiple tasks that can be classified in three categories. First, motion tasks (e.g. balancing the CoG, emulating certain end-effector impedance or template dynamics, etc.) can be written as

$$
\min _{\mathbf{x}}\left\|\ddot{\mathbf{r}}_{i}-\ddot{\mathbf{r}}_{i, \mathrm{des}}\right\|_{2}^{2}
$$

with $\ddot{\mathbf{r}}_{i, \text { des }}$ being the desired task-space acceleration at position $\mathbf{r}_{i}$. Second, joint torque tasks (e.g. achieving specific torque, optimizing for efficiency, etc.) are expressed as

$$
\min _{\mathbf{x}}\left\|\mathbf{W}_{\tau} \boldsymbol{\tau}-\mathbf{b}_{\tau}\right\|_{2}^{2}
$$

with the weighting matrix $\mathbf{W}_{\tau}$ and vector $\mathbf{b}_{\tau}$. And third, contact force tasks (e.g. applying a desired force at a specific location, optimizing the force distribution to minimize the risk of slippage, etc.) are represented as

$$
\min _{\mathbf{x}}\left\|\mathbf{W}_{F} \mathbf{F}_{s}-\mathbf{b}_{F}\right\|_{2}^{2}
$$

with $\mathbf{W}_{F}$ being the force weighting matrix. All these tasks are tightly connected by the EoM (1) which is in fact an equality task

$$
\mathbf{M} \ddot{\mathbf{q}}+\mathbf{h}+\mathbf{J}_{s}^{T} \mathbf{F}_{s}=\mathbf{S}^{T} \boldsymbol{\tau}
$$

linearly depending on generalized accelerations $\ddot{\mathbf{q}}$, contact forces $\mathbf{F}_{s}$, and joint torques $\boldsymbol{\tau}$. To complete the behavior description, the robot has to additionally deal with position limitations $\mathbf{r}_{i}<\mathbf{r}_{i, \text { lim }}$, torque limitations $\boldsymbol{\tau}_{i}<\boldsymbol{\tau}_{i, \text { lim }}$, or contact force limitations $\mathbf{F}_{s, i}<\mathbf{F}_{s, i, l i m}$. Position limitations can be written as $\ddot{\mathbf{r}}_{i}<\ddot{\mathbf{r}}_{i, l i m}$, where $\ddot{\mathbf{r}}_{i, l i m}=f\left(\mathbf{r}_{i}, \dot{\mathbf{r}}_{i}\right)$ is computed from the distance of $\mathbf{r}_{i}$ to the limit $\mathbf{r}_{i, \text { lim }}$ at each instant of time.

In eqs. (13) to (16) the optimization vector $x$ corresponds to a combination of the generalized acceleration $\ddot{\mathbf{q}}$, the joint torques $\tau$, and the contact forces $\mathbf{F}_{s}$. In the following we outline and compare different types of whole body control methodologies that are used to calculate the desired actuator input command $\tau$ as the optimal solution of a set of such minimization problems with different priorities.

Note: To ensure dynamic and kinematic consistency, it is important to choose the EoM and contact constraints as tasks with highest priority. 


\subsection{Full Optimization Vector}

The natural approach introduced by de Lasa and Hertzmann $(2009)$ is to choose

$$
\mathbf{x}^{f}=\left(\begin{array}{c}
\ddot{\mathbf{q}} \\
\boldsymbol{\tau} \\
\mathbf{F}_{s}
\end{array}\right)
$$

as the full stacked vector of all optimization parameters, which yields the following optimization matrices and vectors:

Table 1: Least squares optimization matrices for the full optimization vector $\mathbf{x}^{f}(17)$.

\begin{tabular}{|c|c|c|c|c|c|c|c|}
\hline \multicolumn{2}{|l|}{ EoM (16) } & \multicolumn{2}{|l|}{ motion task 13} & \multicolumn{2}{|l|}{ torque task (14) } & \multicolumn{2}{|l|}{ force task (15) } \\
\hline $\mathbf{A}=\left[\mathbf{M},-\mathbf{S}^{T}, \mathbf{J}_{s}^{T}\right]$ & (18) & $\mathbf{A}=\left[\mathbf{J}_{i}, \mathbf{0}, \mathbf{0}\right]$ & (20) & $\mathbf{A}=\left[\mathbf{0}, \mathbf{W}_{\tau}, \mathbf{0}\right]$ & $(22)$ & $\mathbf{A}=\left[\mathbf{0}, \mathbf{0}, \mathbf{W}_{F}\right]$ & (24) \\
\hline $\mathbf{b}=-\mathbf{h}$ & (19) & $\mathbf{b}=\ddot{\mathbf{r}}_{i, \mathrm{des}}-\dot{\mathbf{J}}_{i} \dot{\mathbf{q}}$ & (21) & $\mathbf{b}=\mathbf{b}_{\tau}$ & (23) & $\mathbf{b}=\mathbf{b}_{F}$ & $(25)$ \\
\hline
\end{tabular}

\subsection{Reduced Optimization Vector}

The EoM (16) represents a linear dependency of generalized accelerations $\ddot{\mathbf{q}}$, joint torques $\boldsymbol{\tau}$, and contact forces $\mathbf{F}_{s}$. Since this task has always highest priority, it is guaranteed that the constraining equality is fulfilled. Hence, the optimization dimensionality can be reduced.

\subsubsection{Acceleration and Torque Optimization}

A well known method from inverse dynamics control with floating base systems (e.g. Mistry et al., 2010) is to use projected system dynamics as illustrated in (3)

$$
\mathbf{P}_{F}(\mathbf{M} \ddot{\mathbf{q}}+\mathbf{h})=\mathbf{P}_{F} \mathbf{S}^{T} \boldsymbol{\tau},
$$

such that the dimensionality of the optimization vector can be reduced to

$$
\mathbf{x}^{r F}=\left(\begin{array}{c}
\ddot{\mathbf{q}} \\
\boldsymbol{\tau}
\end{array}\right)
$$

While there exist different choices for $\mathbf{P}_{F}$ (Righetti et al. 2011a), we apply in the experimental section of this paper the well established QR decomposition of $\mathbf{J}_{s}^{T}=\mathbf{Q}\left[\begin{array}{ll}\mathbf{R}^{T} & \mathbf{0}\end{array}\right]^{T}$ with the orthogonal matrix $\mathbf{Q}^{T}=\mathbf{Q}^{-1}$ and the upper right triangular matrix $\mathbf{R}$. Through the decomposition of $\mathbf{Q}=\left[\begin{array}{ll}\mathbf{Q}_{c} & \mathbf{Q}_{u}\end{array}\right]$ into constrained and unconstrained components, the linear mapping is written as $\mathbf{P}_{F}=\mathbf{Q}_{u}^{T} \in \mathbb{R}^{n_{m} \times n}$. The QR decomposition gives additionally direct access to the contact force

$$
\mathbf{F}_{s}=\mathbf{R}^{-1} \mathbf{Q}_{c}^{T}\left(\mathbf{S}^{T} \boldsymbol{\tau}-(\mathbf{M} \ddot{\mathbf{q}}+\mathbf{h})\right) .
$$

Using this methodology, the optimization procedure results to

Table 2: Least squares optimization matrices for the reduced optimization vector $\mathbf{x}^{r F}$ (27).

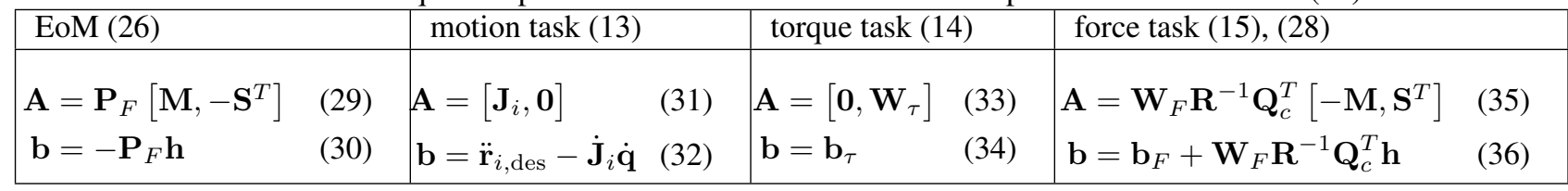

\subsubsection{Acceleration and Force Optimization}

An alternative method, which is similar to the ideas presented in (Herzog et al.), is to eliminate the joint torques as constraint variables. To this end, we use a linear mapping ${ }^{1} \mathbf{P}_{S}$ into the null-space of the actuation matrix $\mathbf{S}$, which yields the Newton-Euler equations of the whole system

$$
\mathbf{P}_{S}\left(\mathbf{M} \ddot{\mathbf{q}}+\mathbf{h}+\mathbf{J}_{s}^{T} \mathbf{F}_{s}\right)=\mathbf{0}
$$

and the corresponding reduced optimization vector

$$
\mathbf{x}^{r S}=\left(\begin{array}{c}
\ddot{\mathbf{q}} \\
\mathbf{F}_{s}
\end{array}\right)
$$

\footnotetext{
${ }^{1}$ In analogy to the discussion for $\mathbf{P}_{F}$, we refer to this as projection
} 
In fact, since $\mathbf{S}=\left[\begin{array}{ll}\mathbf{0}_{\left(n_{r} \times n_{b}\right)} & \mathbf{I}_{\left(n_{r} \times n_{r}\right)}\end{array}\right]$, the simplest possible choice is $\mathbf{P}_{S}=\left[\begin{array}{ll}\mathbf{I}_{\left(n_{b} \times n_{b}\right)} & \mathbf{0}_{\left(n_{b} \times n_{r}\right)}\end{array}\right]$. Given he optimization parameters $\ddot{\mathbf{q}}$ and $\mathbf{F}_{s}$, the joint torques are generated as

$$
\boldsymbol{\tau}=\mathbf{S}\left(\mathbf{M} \ddot{\mathbf{q}}+\mathbf{h}+\mathbf{J}_{s}^{T} \mathbf{F}_{s}\right)
$$

which yields the following optimization tasks:

Table 3: Least squares optimization matrices for the reduced optimization vector $\mathbf{x}^{r S}(38)$.

\begin{tabular}{|ll|l|l|l|l|}
\hline EoM (37) & motion task [13) & torque task [14), (39) & force task (15) \\
\hline $\mathbf{A}=\mathbf{P}_{S}\left[\mathbf{M}, \mathbf{J}_{s}^{T}\right]$ & $(40)$ & $\mathbf{A}=\left[\mathbf{J}_{i}, \mathbf{0}\right]$ & $\mathbf{A}=\mathbf{S}\left[\mathbf{M}, \mathbf{J}_{s}^{T}\right]$ \\
$\mathbf{b}=-\mathbf{P}_{S} \mathbf{h}$ & $(41)$ & $\mathbf{b}=\ddot{\mathbf{r}}_{i, \mathrm{des}}-\dot{\mathbf{J}}_{i} \dot{\mathbf{q}}$ & (43) & $\mathbf{b}=\mathbf{b}_{\tau}-\mathbf{S h}$ & $\mathbf{A}=\left[\mathbf{0}, \mathbf{W}_{F}\right]$ \\
\hline
\end{tabular}

\subsection{Sequential Optimization}

In many application of whole body control techniques, the primary goal is to achieve a desired motion while the optimization in joint torques or contact forces is only considered as a byproduct that allows to lower the energy consumption or to increase stability without changing the motion (e.g. Righetti et al., 2013). This corresponds to splitting the optimization in two subsequent steps with higher priority assigned to the motion tasks.

\subsubsection{Step 1: Kinematic Motion Optimization}

The desired generalized accelerations can be generated using

$$
\mathbf{x}^{q}=\ddot{\mathbf{q}}, \quad \mathbf{A}_{i}^{q}=\mathbf{J}_{i}, \quad \mathbf{b}_{i}^{q}=\ddot{\mathbf{r}}_{i, \mathrm{des}}-\dot{\mathbf{J}}_{i} \dot{\mathbf{q}}
$$

which corresponds to pure kinematic prioritization as already done by Siciliano and Slotine (1991) and many others. By considering the contact constraint $\ddot{\mathbf{r}}_{s}=\mathbf{J}_{s} \ddot{\mathbf{q}}+\dot{\mathbf{J}}_{s} \dot{\mathbf{q}}=\mathbf{0}$ with highest priority, kinematic consistency is ensured.

\subsubsection{Step 2: Inverse Dynamics and Null-Space Opimization}

In the second step, the desired actuator torque can be calculated by inverting the projected equations according to (4), which corresponds to using

$$
\mathbf{x}^{\tau}=\boldsymbol{\tau}, \quad \mathbf{A}_{1}^{\tau}=\mathbf{P}_{F} \mathbf{S}^{T}, \quad \mathbf{b}_{1}^{\tau}=\mathbf{P}_{F}(\mathbf{M} \ddot{\mathbf{q}}+\mathbf{h})
$$

as task with highest priority for torque optimization. If there are linearly depending contact conditions respectively internal force directions, $\mathbf{A}_{1}^{\tau}$ has an associated null-space projector $\mathbf{N}_{P S}$ which allows for additional torque

$$
\min _{\boldsymbol{\tau}}\left\|\mathbf{W}_{\tau} \boldsymbol{\tau}-\mathbf{b}_{\tau}\right\|_{2}^{2}
$$

or force optimization, which can be transformed using [28) into a least squares problem that is linear in joint torques $\tau$ :

$$
\min _{\mathbf{F}_{s}}\left\|\mathbf{W}_{F} \mathbf{F}_{s}-\mathbf{b}_{F}\right\|_{2}^{2} \stackrel{28}{=} \min _{\boldsymbol{\tau}}\left\|\mathbf{W}_{F} \mathbf{R}^{-1} \mathbf{Q}_{c}^{T} \mathbf{S}^{T} \boldsymbol{\tau}-\left(\mathbf{b}_{F}+\mathbf{W}_{F} \mathbf{R}^{-1} \mathbf{Q}_{c}^{T}(\mathbf{M} \ddot{\mathbf{q}}+\mathbf{h})\right)\right\|_{2}^{2} .
$$

\begin{tabular}{|c|c|c|c|c|c|c|}
\hline EoM (49) & & motion task (48) & & torque task 50 & force task (51) & \\
\hline $\mathbf{x}^{\tau}=\tau$ & & $\mathbf{x}^{q}=\ddot{\mathbf{q}}$ & & $\mathbf{x}^{\tau}=\tau$ & $\mathbf{x}^{\tau}=\boldsymbol{\tau}$ & \\
\hline $\mathbf{A}^{\tau}=\mathbf{P}_{F} \mathbf{S}^{T}$ & $(52)$ & $\mathbf{A}^{q}=\mathbf{J}_{i}$ & $(54)$ & $\mathbf{A}^{\tau}=\mathbf{W}_{\tau}$ & $\mathbf{A}^{\tau}=\mathbf{W}_{F} \mathbf{R}^{-1} \mathbf{Q}_{c}^{T} \mathbf{S}^{T}$ & $(58)$ \\
\hline $\mathbf{b}^{\tau}=\mathbf{P}_{F}(\mathbf{M} \ddot{\mathbf{q}}+\mathbf{h})$ & (53) & $\mathbf{b}^{q}=\ddot{\mathbf{r}}_{i, \mathrm{des}}-\dot{\mathbf{J}}_{i} \dot{\mathbf{q}}$ & $(55)$ & $\mathbf{b}^{\tau}=\mathbf{b}_{\tau}$ & $\mathbf{b}^{\tau}=\mathbf{b}_{F}+\mathbf{W}_{F} \mathbf{R}^{-1} \mathbf{Q}_{c}^{T}(\mathbf{M} \ddot{\mathbf{q}}+\mathbf{h})$ & $(59)$ \\
\hline
\end{tabular}

In summary, this results in the following least squares problems that are solved in two steps:

Table 4: Least squares optimization matrices for sequential optimization of the desired motion $\mathrm{x}^{q}$ and torque $\mathrm{x}^{\boldsymbol{\tau}}$.

In (Hutter. 2013, chp. 5.4.3) we show how this method is a general formulation of the weighted pseudo-inversion presented in (Righetti et al., 2013) to minimize a quadratic cost in joint torques and contact forces. As an advantage, the method here allows to include multiple tasks in the support null-space as e.g., required to additionally limit the joint torques (see Section 5.1.3.

Note: This second step can be equally stated by using the projected EoM $[37)$ with $\mathbf{x}^{F}=\mathbf{F}_{s}$ as optimization variable. To keep the paper compact, this method is omitted. 


\subsection{Summary}

The full (Section 4.1) and reduced (Section 4.2) optimization have the advantage to guarantee kinematic and dynamic consistency as $\mathbf{A}_{1}$ described in (18), 29] and (40) has no rank deficiency due to the positive definite property of $\mathbf{M}$. Moreover, the reduced methodology offers the same functionality with a lower dimension of the optimization. As a drawback, both methods include the mass matrix $\mathbf{M}$ in the optimization matrix $\mathbf{A}$ which has to be inverted to solve the prioritized least-square problem (see (11)). Depending on the system properties, this can lead to numerical problems as M can be badly conditioned (very low inertia at end-effectors but high mass at the base (see also Featherstone, 2004)). However, this was never a problem for the simulations and experiments presented in Section 5

The sequential approach has the advantage of being a pure kinematic construct (cf. Righetti et al. 2011a). As a shortcoming, dynamic consistency cannot be guaranteed since $\mathbf{A}_{i}$ defined in (52) is rank deficient if the system is trulyunderactuated. This implies that the robot has partially uncontrollable dynamics. To give an example, a quadrupedal robot with two feet in ground contact leaves the dynamics uncontrollable in one dimension as the angular momentum around the line of support cannot be changed although the desired accelerations as calculated by the kinematic prioritization in step 1 may require so. Hence, this method is only applicable for static maneuvers.

\section{Results}

The presented approaches were tested in a realtime $\mathrm{C} / \mathrm{C}++$ simulation and control environment $($ Hutter et al. 2012a. Schaal, 2009) and experimentally validated on StarlETH (Hutter et al., 2012a) autonomously operated with onboard state estimation (Bloesch et al., 2012). The inverse dynamics control framework was designed using analytical expressions for the system kinematics and dynamics (Hutter et al., 2011a) based on a detailed CAD design. All our experiments use pure task-space inverse dynamics control without any (low-gain) joint position regulation (cf. Buchli et al., 2009). Due to its compact formulation, the analytical iterative null-space projection (Section 3.1) is applied to resolve the least squares task hierarchy.

We conducted two sets of experiments with a different focus. In Section 5.1, static walking is used as a showcase to investigate joint torque and contact force optimization. Multi contact situations ( 3 or 4 feet are always in ground contact) make the system overconstrained and open different possibilities to optimize the load distribution. In Section 5.2, the experiments are extended to dynamic trotting gaits whereby the system is truly-underactuated In this part we focus on the motion control side and implement an intuitive strategy with the desired task-space dynamics represented in different coordinate systems. Given the properties of the full (Section 4.1), reduced (Section 4.2), and sequential approach (Section 4.3), we apply the sequential method (summarized in Table 4) for static locomotion (Sim/Exp 1-9) and the reduced method (summarized in Table 2) for dynamic experiments (Sim/Exp 10-12). All results are illustrated in Extension 1.

\subsection{Static walking}

The application of a static crawling gait ensures that the overall CoG is kept over the support polygon at any point of time. To this end we use a basic trajectory planner. In fact, way points for the moving foot and the base location of two successive steps are planned ahead to minimize the body motion while guaranteeing a certain safety margin defined as distance between base and support polygon. Thereby, the robot continuously executes a stereotypic footfall pattern (McGhee et al. 1968) left-hind (LH), left-front (LF), right-hind (RH), right-front (RF).

We conduct a set of simulations ( $\mathrm{Sim})$ and real hardware experiments (Exp) that are based on the same task-space motion optimization. For step 1, the motion optimization (Section 4.3.1, the support-constraint with $\mathbf{A}_{1}^{q}=\mathbf{J}_{s}$ and $\mathbf{b}_{1}^{q}=-\dot{\mathbf{J}}_{s} \dot{\mathbf{q}}$ has the highest priority $P_{1}^{q}$. The base position and orientation is combined in a single task of lower priority $P_{2}^{q}$ with $\mathbf{A}_{2}^{q}=\left[\begin{array}{ll}\mathbf{J}_{B}^{T} & \mathbf{J}_{R}^{T}\end{array}\right]^{T}$ and $\ddot{\mathbf{r}}_{2, \text { des }}=\mathbf{k}_{p}\left(\mathbf{r}_{2, \text { des }}-\mathbf{r}_{2}\right)+\mathbf{k}_{d}\left(\dot{\mathbf{r}}_{2, \text { des }}-\dot{\mathbf{r}}_{2}\right) \in \mathbb{R}^{n_{b}}$. Thereby, $\mathbf{J}_{B}=\frac{\partial \mathbf{r}_{\text {base }}}{\partial \mathbf{q}}$ represents the translational base Jacobian, $\mathbf{J}_{R}=\frac{\partial \boldsymbol{\Omega}}{\partial \dot{\mathbf{q}}}$ the rotational Jacobian, and $\boldsymbol{\Omega}$ the main body rotation speed. The base orientation and rotation speed are always controlled to zero.

During the shifting as well as the stepping sequence, at least $n_{c p} \geq 3$ points are in contact with the ground in a non-singular configuration. Using Definition 2 the system is $n_{c}=3 n_{c p}-n_{b}$ times overconstrained Taking for step 2 (Section 4.3.2) the constrained dynamics task (49) as highest priority $P_{1}^{\tau}$ there remains $n_{c}$ internal directions for contact force respectively joint torque optimization strategies with lower priority $\left(P_{1}^{\tau}>P_{2}^{\tau}>\ldots\right)$ without impairing the motion. The different sets of optimization objectives summarized in Table 5 are compared with respect to energy efficiency and robustness. As a measure for efficiency of electrically driven robots, the most commonly used objective function is the integral of the square of actuator torques (Remy et al., 2012)

$$
E_{\tau}=\int \boldsymbol{\tau}^{T} \boldsymbol{\tau} \mathrm{d} t
$$

To quantify the risk of slippage, we calculate the mean of the relation between tangential and normal contact force

$$
\bar{\mu}=\operatorname{mean}\left(\frac{F_{\text {tangential }}(t)}{F_{\text {normal }}(t)}\right)
$$


Table 5: Static walking experiments with different optimization objectives

\begin{tabular}{|c|c|c|c|c|c|c|c|c|c|}
\hline units & $P_{1}^{\tau}$ & $P_{2}^{\tau}$ & $P_{3}^{\tau}$ & $\begin{array}{c}E_{\tau} \\
{\left[\mathrm{N}^{2} \mathrm{~m}^{2} \mathrm{~s}\right]}\end{array}$ & $\begin{array}{c}\bar{\mu} \\
{[-]}\end{array}$ & $\begin{array}{c}\tau_{K F E}^{\max } \\
{[\mathrm{Nm}]}\end{array}$ & $\begin{array}{c}\Delta \mathbf{r}_{\text {sim-exp }} \\
{[\mathrm{mm}]}\end{array}$ & $\begin{array}{l}\overline{\overline{\Delta r}_{s i m}} \\
{[\mathrm{~mm}]}\end{array}$ & $\begin{array}{c}\overline{\Delta r}_{\text {exp }} \\
{[\mathrm{mm}]}\end{array}$ \\
\hline Sim1 & inv dyn* & $\tau \min ^{\ddagger}$ & - & 3985 & 0.27 & 12.2 & \multirow{2}{*}{11.1} & 1.4 & \multirow[b]{2}{*}{4.2} \\
\hline Exp1 & inv dyn* & $\tau \min ^{\ddagger}$ & - & 4701 & 0.20 & 12.4 & & & \\
\hline $\operatorname{Sim} 2$ & inv dyn* & $\mathbf{F}_{s} \min ^{\S}$ & - & 5730 & 0.04 & 16.0 & \multirow{2}{*}{6.6} & 0.7 & \multirow[b]{2}{*}{3.2} \\
\hline Exp2 & inv dyn* & $\mathbf{F}_{s} \min ^{\S}$ & - & 5544 & 0.04 & 13.7 & & & \\
\hline $\operatorname{Sim} 3$ & inv dyn* & $\tau \lim ^{\dagger}$ & $\tau \min ^{\ddagger}$ & 4034 & 0.29 & 10.0 & \multirow{2}{*}{11.3} & 0.7 & \multirow[b]{2}{*}{3.7} \\
\hline Exp3 & inv dyn* & $\tau \lim ^{\dagger}$ & $\tau \min ^{\ddagger}$ & 4557 & 0.24 & 10.4 & & & \\
\hline $\operatorname{Sim} 4$ & inv dyn* & $\tau \lim ^{\dagger}$ & $\mathbf{F}_{s} \min ^{\ddagger}$ & 5502 & 0.10 & 10.0 & \multirow{2}{*}{7.0} & 1.1 & \multirow[b]{2}{*}{4.5} \\
\hline Exp4 & inv dyn* & $\tau \lim ^{\dagger}$ & $\mathbf{F}_{s} \min ^{\S}$ & 5485 & 0.07 & 10.6 & & & \\
\hline \multicolumn{3}{|c|}{$\begin{array}{l}\text { *inv dyn: } \\
{ }^{\dagger} \tau \lim \left(\tau_{K F E_{L F}}<10 \mathrm{Nm}\right): \\
{ }^{\ddagger} \tau \min \left(\mathbf{W}_{\tau}=\mathbf{I}, \mathbf{b}_{\tau}=\mathbf{0}\right): \\
\S \mathbf{F}_{s} \min \left(\mathbf{W}_{F}=\mathbf{I}, \mathbf{b}_{F}=\mathbf{0}\right)\end{array}$} & $\begin{aligned} \mathbf{A}_{i}^{\tau} & = \\
\mathbf{A}_{i}^{\tau} & = \\
\mathbf{A}_{i}^{\tau} & = \\
\mathbf{A}_{i}^{\tau} & =\end{aligned}$ & $\begin{array}{l}\mathbf{Q}_{u}^{T} \mathbf{S}^{T}, \\
0 \quad 0 \quad 1 \\
\mathbf{W}_{\tau}, \\
\mathbf{R}^{-1} \mathbf{Q}_{c}^{T} \mathbf{S}^{T}\end{array}$ & 0 & $0]$, & $\begin{aligned} \mathbf{b}_{i}^{\tau} & =\mathbf{Q}_{u}^{T}(\mathbf{M} \dot{c} \\
\mathbf{b}_{i}^{\tau} & =10 \\
\mathbf{b}_{i}^{\tau} & =\mathbf{0} \\
\mathbf{b}_{i}^{\tau} & =\mathbf{R}^{-1} \mathbf{Q}_{c}^{T}\end{aligned}$ & $\mathbf{M} \ddot{\mathbf{q}}+\mathbf{h})$ & \\
\hline
\end{tabular}

The smaller this fraction, the lower the risk of slippage at the evaluated contact point. Since there are no precise contact force sensors incorporated, these forces are estimated in the experiments by (28) based on joint torque, encoder, and IMU signals. Due to an accurate model and precise torque measurements, this approximation yields quite precise results as validated by Fankhauser et al. (2012).

Simulation and experimental measurements agree well in the actual base position and orientation. For illustration, we evaluated the average absolute distance between the simulation and the experiment as $\Delta \mathbf{r}_{\text {sim }_{i}-\exp _{i}}=$ mean $\left|\mathbf{r}_{\text {sim }_{i}}(t)-\mathbf{r}_{\text {exp }_{i}}(t)\right|$. The maximal offset of $11 \mathrm{~mm}$ over the full recording time of $15 \mathrm{~s}$ mainly origins from the time shift that is accumulated, since the gait cycle is not time but phase synchronized. Considering only a single step, the error remains below $5 \mathrm{~mm}$. Second, we investigated the influence of the different optimization strategies on position tracking, which ideally should also be zero. To this end, the average absolute distance error $\overline{\Delta \mathbf{r}}_{i}=$ mean $\left|\mathbf{r}_{i}(t)-\overline{\mathbf{r}}(t)\right|$ of each $\left(n_{e}=4\right)$ simulation and experiment $\mathbf{r}_{i}(t)$ to the mean $\overline{\mathbf{r}}(t)=\frac{1}{n_{e}} \sum_{i=1}^{n_{e}} \mathbf{r}_{i}(t)$ is computed for simulations and experiments independently. With absolute values of less than $1.4 \mathrm{~mm}$ and $4.5 \mathrm{~mm}$ respectively, the robot motion barely differs. Keep in mind that the real world experiments are subject to different model inaccuracies and external disturbances. The results are summarized in Table 5 and discussed in detail subsequently.

\subsubsection{Actuator Efficiency Optimization (Exp1, Sim1)}

The energy consumption is optimized by applying the least squares problem 50 with $\mathbf{A}_{2}^{\tau}=\mathbf{W}_{\tau}=\mathbf{I}$ and $\mathbf{b}_{2}^{\tau}=\mathbf{b}_{\tau}=\mathbf{0}$ with priority $P_{2}^{\tau}$. With this method, a walking sequence of 3 full cycles ( 3 steps with each leg) corresponds to a cost of $E_{\tau}^{\text {sim }}=3985 \mathrm{~N}^{2} \mathrm{~m}^{2} \mathrm{~s}$ in simulations and $E_{\tau}^{\text {exp }}=4701 \mathrm{~N}^{2} \mathrm{~m}^{2} \mathrm{~s}$ in experiments. The comparably large difference of the energy consumption can be explained by modeling inaccuracies (simulation and controller are only based on CAD data) and imperfect torque control performance of the SEA (actuator is not modeled in the simulation). Analyzing the contact forces shows that the mean required friction coefficients are $\bar{\mu}^{\text {sim }}=0.27$ and $\bar{\mu}^{\text {exp }}=0.20$ correspondingly.

\subsubsection{Contact Force Optimization (Exp2, Sim2)}

Minimizing the total force is equivalent to removing all internal forces that do actually not contribute the net force acting on the ground. This is similar to work that was done based on static virtual model control (Zhou et al. 2000) where the pseudo-inversion distributes the virtual forces onto support forces such that they are least square minimal. In the presented framework, force minimization (51) is achieved by selecting $\mathbf{W}_{F}=\mathbf{I}$ and $\mathbf{b}_{F}=\mathbf{0}$, respectively $\mathbf{A}_{2}^{\tau}=\mathbf{R}^{-1} \mathbf{Q}_{c}^{T} \mathbf{S}^{T}$ and $\mathbf{b}_{2}^{\tau}=\mathbf{R}^{-1} \mathbf{Q}_{c}^{T}(\mathbf{M} \ddot{\mathbf{q}}+\mathbf{h})$ (see Table 4 ). While the tangential contact forces are significantly reduced $\left(\bar{\mu}^{\text {sim }}=0.04\right.$, $\left.\bar{\mu}^{e x p}=0.04\right)$, the actuation cost $(60)$ of $E_{\tau}^{s i m}=5730 \mathrm{~N}^{2} \mathrm{~m}^{2} \mathrm{~s}$ and $E_{\tau}^{\text {exp }}=5544 \mathrm{~N}^{2} \mathrm{~m}^{2} \mathrm{~s}$ are increased.

\subsubsection{Joint Torque Limitation (Exp3/4, Sim3/4/5)}

A simple method to account for actuator torque limitations $\left|\tau_{i}\right|<\tau_{\text {sat }}$ is to tackle these inequality constraints as outlined in Section 3.2. Limitations are generally disabled, but as soon as torque limitations are violated, a high priority task with a unit entry at the corresponding location $\mathbf{A}_{2}^{\tau}=\mathbf{W}_{\tau}=\left[\begin{array}{lllllll}0 & 0 & 1 & 0 & \ldots & 0\end{array}\right]$ and $\mathbf{b}_{2}^{\tau}=\mathbf{b}_{\tau}= \pm \tau_{\text {sat }}$ is introduced and all other tasks are shifted to lower priorities. If multiple joints reach torque limitations simultaneously, the corresponding tasks get stacked at the same priority. The procedure ensures that the generated torque profiles meet all actuator limitations and that non-saturated actuators take more load to still achieve the desired acceleration. StarlETH can perform the same walking maneuvers as in the previous experiments but with (arbitrarily) limited knee flexion/extension (KFE) joint torque to $10 \mathrm{Nm}$. As summarized in Table 5 , the energetic consumption remains more or less equal if the maximal knee torque is limited. However, the limitation slightly increases the risk of slipping since the contact force cannot be aligned as well as 


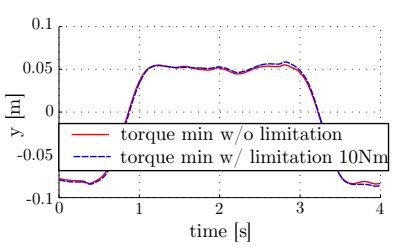

(a) Main body position

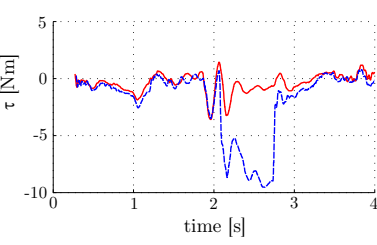

(b) LF HFE torque

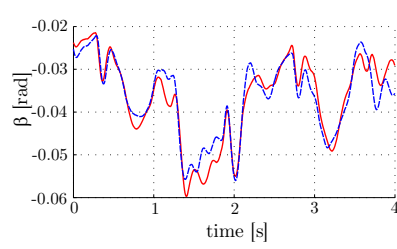

(c) Main body orientation

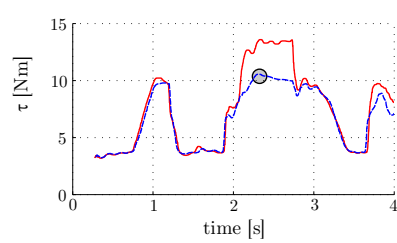

(d) LF KFE torque

Figure 3: A comparison of experimental results with (blue dashed, Exp3) and without (red solid, Exp1) joint torque limitations show very good matching in terms of motion (a,c). During the phase where $\tau_{K F E}$ is saturated (d), HFE (and others) takes over part of the load (b).

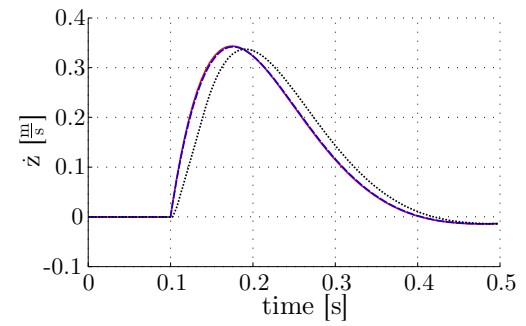

(a) Vertical main body velocity

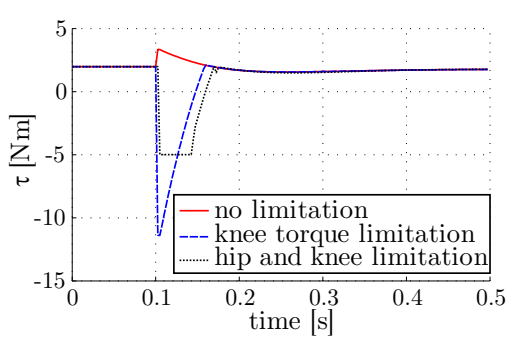

(b) LF HFE torque

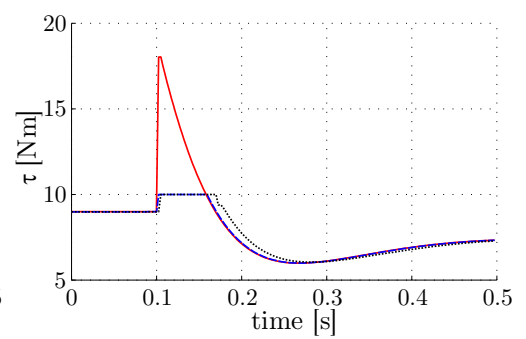

(c) LF KFE torque

Figure 4: (Sim5): As long as only $\tau_{K F E}$ is saturated, the desired motion of a vertical step input on the main body position is perfectly executed since the hip (mainly HFE) is taking over part of the load (blue dashed). If the $\tau_{H F E}$ saturates as well, the desired motion cannot be followed anymore (black dotted).

without torque saturation. As depicted in Figures 3(a) and 3(c), the motion is almost equal for Expl and Exp3 independent of the joint torque distribution. At the same time, the knee torque (Figure 3(d)) can be limited to $10 \mathrm{Nm}$ : in this region, mainly hip flexion/extension (HFE) of the same leg but also other joints take part of the load (Figure 3(b)). Similar results but only in simulations have been presented by ? using a numerical QP solver. Violations of the $10 \mathrm{Nm}$ boundary (gray circle, Figure 3(d) result from torque tracking errors at the SEA, which has non-zero output impedance if the joint is moving (Hutter et al. 2011b).

In case too many joints saturate at the same moment such that there is no feasible solution $\tau$ to achieve the desired dynamics without violating the constraining task (i.e., the equality task $P_{2}^{\tau}$ cannot be fulfilled), the torques $\tau$ satisfy the saturation limitations only as good as possible. However, by applying the reduced approach (Section 4.2), we can assign higher priority to torque limitations than to motion execution. To this end, the EoM is selected as first priority, the contact constraint as second priority, the torque saturation task as third priority, the main body motion as fourth priority, and the torque minimization as fifth priority. This is illustrated in Figure 4 by a vertical position step command signal applied to the body height (Sim5): As long as only KFE is saturated, the desired motion is perfectly executed since the hip (mainly HFE) is taking part of the load (blue dashed). If the HFE torque is limited as well, the desired motion cannot be followed anymore (black dotted) and the task with fourth priority is violated.

\subsubsection{Joint Position Limitation (Exp6, Exp7)}

In addition to torque saturation, most robots suffer from a limited joint or task-space range of operation. This is usually handled by soft limitations when planning the desired motion. However, joint range limitations should also be ensured if the actual motion is significantly offset form the desired motion, e.g. by external disturbances. This happens in particular when regulating the robot with low feedback gains to emulate a compliant behavior. As a solution, we propose to include the position limitation as inequality constraints in the motion controller (Section 3.2). As a showcase sce-

Table 6: Motion task priorities for horizontal main body shifting (Exp 6). In Exp7, the priorities of task 4 and 5 are switched

\begin{tabular}{ccccc} 
Priority & Dimension & reference signal & $\mathbf{A}_{i}^{q}(54)$ & $\mathbf{b}_{i}^{q}(55)$ \\
\hline$P_{1}^{q}$ & changing & support-constraint & ${ }_{I} \mathbf{J}_{s}$ & ${ }_{I} \dot{\mathbf{J}}_{s} \dot{\mathbf{q}}$ \\
$P_{2}^{q}$ & changing & joint angle limitation & $\mathbf{S}_{l i m}$ & $\mathbf{S}_{l i m}\left(\mathbf{k}_{p}^{l i m}\left(\mathbf{q}_{l i m}-\mathbf{q}\right)-\mathbf{k}_{d}^{l i m} \dot{\mathbf{q}}\right)$ \\
$P_{3}^{q}$ & 2 & body pos ${ }_{I} \mathbf{r}_{x y}$ & $\mathbf{S}_{x y}$ & $\mathbf{k}_{p}^{x y}\left({ }_{I} \mathbf{r}_{\mathrm{des}}-{ }_{I} \mathbf{r}\right)+\mathbf{k}_{d}^{x y}\left({ }_{I} \dot{\mathbf{r}}_{\mathrm{des}}-{ }_{I} \dot{\mathbf{r}}\right)$ \\
$P_{4}^{q}$ & 1 & ${\text { body height }{ }_{I} z}_{{ }^{2}}$ & $\mathbf{S}_{z I} \mathbf{J}$ & $k_{p}^{z}\left({ }_{I} z_{\mathrm{des}}-{ }_{I} z\right)+k_{d}^{z}\left({ }_{I} \dot{z}_{\mathrm{des}}-{ }_{I} \dot{z}\right)$ \\
$P_{5}^{q}$ & 3 & body orientation $_{B} \boldsymbol{\varphi}$ & ${ }_{I} \mathbf{J}_{R}$ & $\mathbf{k}_{p}^{\varphi}\left({ }_{I} \boldsymbol{\varphi}_{\mathrm{des}}-{ }_{I} \boldsymbol{\varphi}\right)+\mathbf{k}_{d}^{\varphi}\left({ }_{I} \dot{\boldsymbol{\varphi}}_{\mathrm{des}}-{ }_{I} \dot{\boldsymbol{\varphi}}\right)$ \\
\hline
\end{tabular}




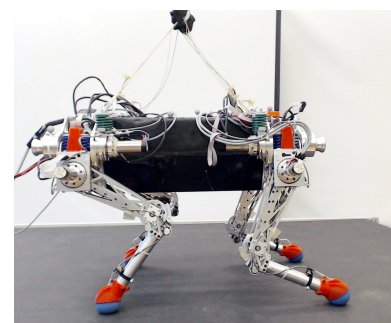

(a) Start configuration

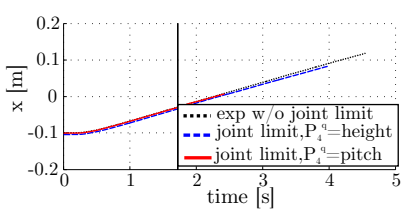

(e) Body forward position

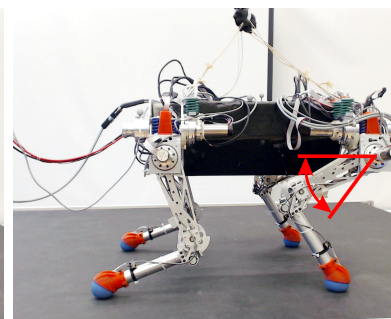

(b) No joint limitation

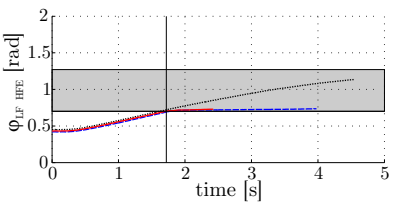

(f) LF HFE joint angle

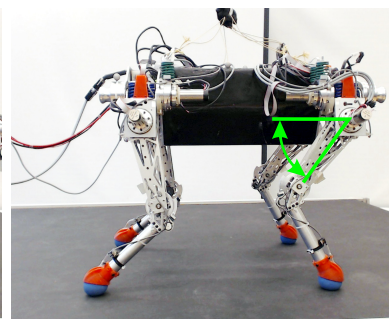

(c) Height compensation

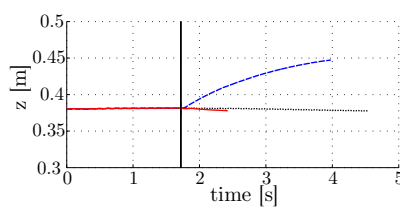

(g) Body height

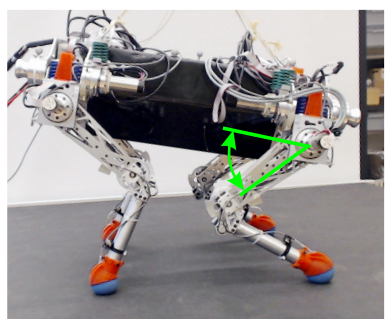

(d) Pitch compensation

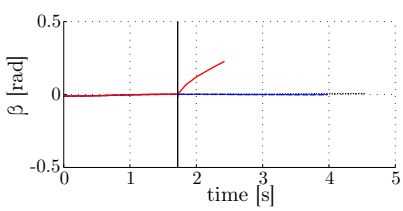

(h) Body pitch angle

Figure 5: The presented framework compensates for joint angle limitations (e.g. $\varphi_{H F E}$ ) while shifting the main body forward. Its motion is distorted either by lifting the main body upward (Exp6, low priority in z posture, (c)) or by tilting the main body forward (Exp7, low priority for body pitch angle, (d)).

nario, a simple base shift is commanded in forward direction. At the same time, the maximal HFE angle is restricted to $0.9 \mathrm{rad}$ from the main body $\mathrm{x}$-axis as indicated in Figure 5. This limitation is arbitrarily chosen to demonstrate the working principle when separating main body position and orientation tasks into different priorities (Table 6). In Exp6, the motion optimization (Section 4.3.1. step 1 of sequential approach) is performed like in the previous examples with the support constraint at highest priority $P_{1}^{q}$. The third priority task $P_{3}^{q}$ attributes to regulate the body position in xyplane (stability) with $\mathbf{S}_{x y}$ representing the corresponding selection matrix. As $P_{4}^{q}$, the body orientation is aligned with the environment while $P_{5}^{q}$ keeps the ground clearance constant. In $\operatorname{Exp} 7$, the order of $P_{4}^{q}$ and $P_{5}^{q}$ is changed such that ground clearance becomes more important than the body orientation. Body shifting in forward direction, while keeping body height and orientation constant, is first conducted without considering the limits (Figure 5(b)). Thereby, the hip joint angle limitation is violated (Figure 5(f), black dotted). This can be avoided by activating the limitation tasks as done in Exp6 (Figure 5(c)) and Exp7 (Figure 5(d)). As soon as the limitation in HFE is reached, a task with $P_{2}^{q}$ is activated that ensures that the angle is not growing anymore in this direction, respectively that the corresponding joint acceleration $\mathbf{S}_{l i m} \ddot{\mathbf{q}}=\mathbf{S}_{l i m}\left(\mathbf{k}_{p}^{l i m}\left(\mathbf{q}_{l i m}-\mathbf{q}\right)-\mathbf{k}_{d}^{l i m} \dot{\mathbf{q}}\right)$ is pushing away from the soft joint angle limitation. The parameters $\mathbf{k}_{p}^{l i m}$ and $\mathbf{k}_{d}^{l i m}$ represent the virtual constraint stiffness and damping, $\mathbf{S}_{\text {lim }}$ is the selection matrix of the joint that violate a position constraint. It can be observed that HFE does not exceed the limitation (Figure 5(f) but that the body motion is distorted by violating the lowest priority task. The robot starts to increase the body clearance (Exp6, Figures 5(c) and 5(g)) or pitching angle (Exp7, Figures 5(d) and 5(h) such that the joint limitation can be avoided.

\subsubsection{Terrain Dependent Optimization $(\operatorname{Exp} 8)$}

When it comes to walking in non-flat terrain, stability at the contact points can only be guaranteed if the contact forces are within the local friction cone at all time. To keep the tangential forces as small as possible, the contact forces are aligned with the local surface normal directions (Hutter et al. 2012b) by a force optimization task. To this end, the force weighting matrix (15) is selected as $\mathbf{W}_{F}=$ blockdiagonal $\left(\alpha_{i} \mathbf{t}_{i}^{T}\right)$ with $\mathbf{t}_{i} \in \mathbb{R}^{3 \times 2}$ being the local tangential plane at each contact point. We tested this in static, in-place walking on a half-cylindrical shaped surface with known geometry (Figure 6(a)). To quantify slippage, we measured the feet positions by post-processing the recorded movie using a color blob-tracker for the ball feet. As indicated in Figure 6(b) foot point slippage can be reduced by this contact force alignment. The method works robust and requires only binary contact switch detection without precise force measurement. As long as the estimated ground surface normal directions are not completely wrong (i.e., better than the global vertical direction), tangential contact forces and hence the risk of slippage are lowered. Hutter et al. (2012b) compares this approach including local surface tangential planes with a sequential QP solver that directly minimizes the local friction coefficient. For a static walking gait, the nonlinear optimization results were only slightly better as the proposed least squares solution but in return required significantly more computational power.

\subsubsection{Smooth Contact Force Distribution (Sim9)}

A change in the contact situation entails a discrete change in the support Jacobian $\mathbf{J}_{s}$. As a consequence, applying inverse dynamics leads to undesired discontinuities in the actuator torques $\tau$ and contact forces $\mathbf{F}_{s}$. To make these changes smooth, we propose an interpolation method between two subsequent contact situations. 


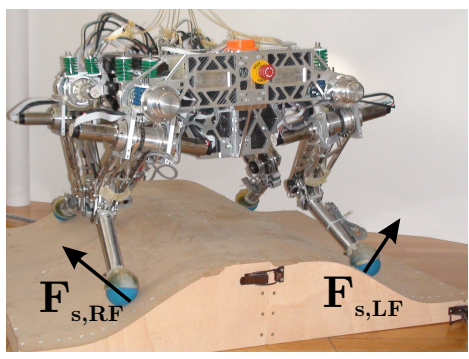

(a) StarlETH on curved surface

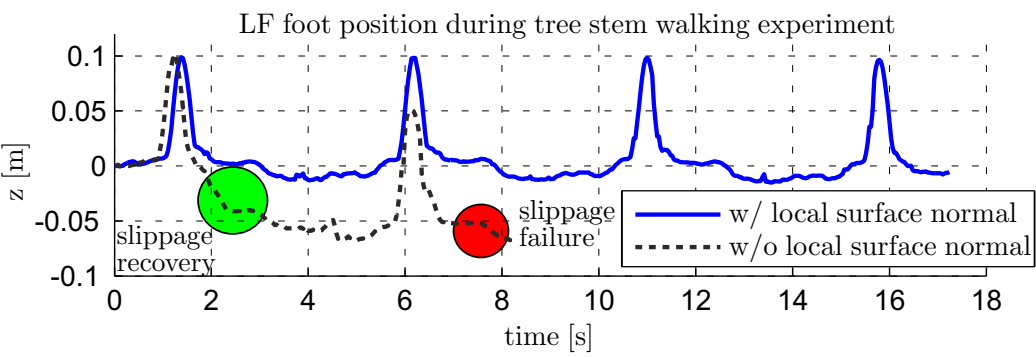

(b) Foot position

Figure 6: Static walking experiment with StarlETH on a half-cylinder surface (a). Aligning the contact forces with the local surface normal direction by applying internal forces allows slippage free walking (blue solid). Considering the surface as flat ground leads to failure after the first step (black dashed).

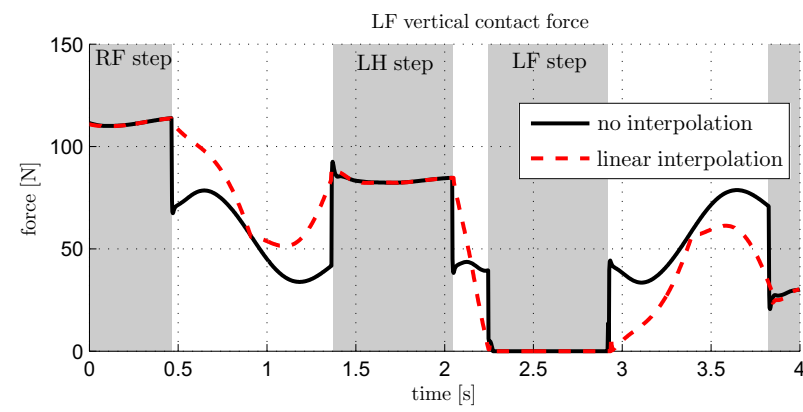

(a) LF Contact Force

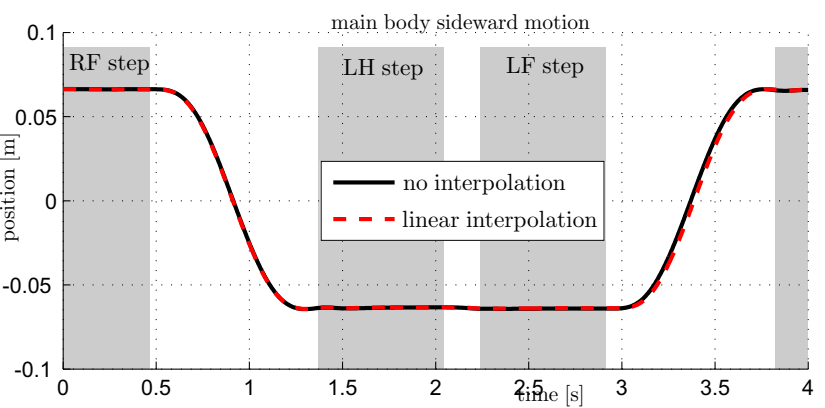

(b) y Body position

Figure 7: Contact force interpolation leads to smooth switches of the contact configurations. The resulting torque trajectories can be followed even by rather low bandwidth actuators as employed in StarlETH.

In case of static walking, transitions occur always between three and four contact point situations in both directions. First, the original contact force problem is solved to generate the optimal load distribution $\mathbf{F}^{\text {opt }}$ for four contact points. Subsequently, the desired contact force at the changing contact is (linearly) scaled to $\mathbf{F}_{j}=w \mathbf{F}_{j}^{o p t}$ as a function of the interpolation parameter $w \in\left[\begin{array}{ll}0 & 1\end{array}\right]$ which is determined by the gait phase or time. Increasing $w$ from 0 to 1 corresponds to loading a leg, decreasing to unloading. Second, the optimization problem is solved again with an additional high priority task $\left(\mathbf{A}_{2}^{\tau}, \mathbf{b}_{2}^{\tau}\right)$ with $\mathbf{W}_{F}=\mathbf{S}_{j}$ and $\mathbf{b}_{F}=\mathbf{F}_{j}$, whereby $\mathbf{S}_{j}$ corresponds to the selection matrix of the interpolated contact force $\mathbf{F}_{j}$. As a result, the load distribution is smoothly transitioned between loading four $\left(\mathbf{F}_{j}=\mathbf{F}_{j}^{o p t}\right)$ or only three $\left(\mathbf{F}_{j}=\mathbf{0}\right)$ legs. This method differs from approaches used by Dietrich et al. (2012) in a way that the interpolation directly shapes the contact forces and not the corresponding null-space projectors $\mathbf{N}_{i}$. As indicated in Figure 7(a), the forces and hence also the joint torque transitions are smooth between the different support configurations. Again, the body motion is not affected by this change in load distribution (Figure 7(b)).
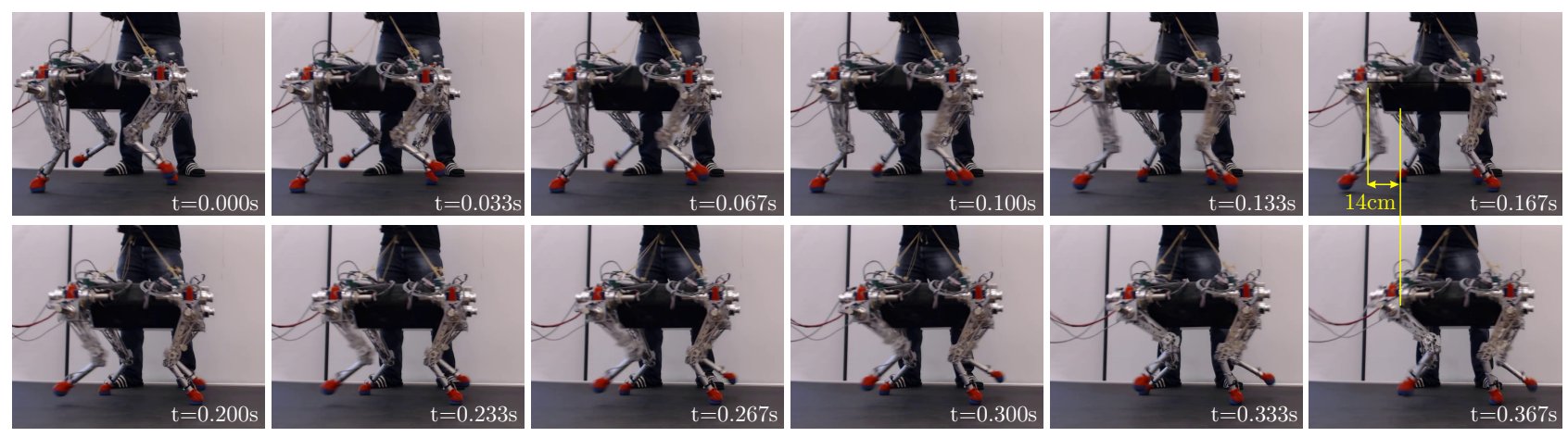

Figure 8: Trot series with a forward speed of approximately $0.7 \mathrm{~m} / \mathrm{s}$ respectively 1.5 bodylength $/ \mathrm{s}$ 
Table 7: Motion task priorities for dynamic locomotion with StarlETH.

\begin{tabular}{|c|c|c|c|c|}
\hline Priority & Dimension & reference signal & $\mathbf{A}_{i}^{c} 31$ & $\mathbf{b}_{i}$ (32) \\
\hline$P_{1}^{q}$ & changing & support constraint & {$\left[\begin{array}{ll}{ }_{I} \mathbf{J}_{s} & \mathbf{0}\end{array}\right]$} & $-{ }_{I} \dot{\mathbf{J}}_{s} \dot{\mathbf{q}}$ \\
\hline$P_{2}^{q}$ & 1 & body height $_{I} z$ & {$\left[\begin{array}{ll}\mathbf{S}_{z I} \mathbf{J} & \mathbf{0}\end{array}\right]$} & $k_{p}^{z}\left({ }_{I} z_{d e s}-{ }_{I} z\right)+k_{d}^{z}\left({ }_{I} \dot{z}_{d e s}-{ }_{I} \dot{z}\right)$ \\
\hline$P_{3}^{q}$ & 2 & body roll, pitch ${ }_{B} \varphi$ & {$\left[\begin{array}{ll}\mathbf{S}_{\varphi B} \mathbf{J}_{R} & 0\end{array}\right]$} & $\mathbf{k}_{p}^{\varphi}\left({ }_{B} \boldsymbol{\varphi}_{\text {des }}-{ }_{B} \boldsymbol{\varphi}\right)+\mathbf{k}_{d}^{\varphi}\left({ }_{B} \dot{\boldsymbol{\varphi}}_{\text {des }}-{ }_{B} \dot{\boldsymbol{\varphi}}\right)-\mathbf{S}_{\varphi B} \dot{\mathbf{J}}_{R} \dot{\mathbf{q}}$ \\
\hline$P_{4}^{q}$ & 1 & body turning rate ${ }_{I} \dot{\psi}$ & {$\left[\mathbf{S}_{\psi I} \mathbf{J}_{R}\right.$} & $k_{d}^{\psi}\left({ }_{I} \dot{\psi}_{\text {des }}-{ }_{I} \dot{\psi}\right)-\mathbf{S}_{\psi I} \dot{\mathbf{J}}_{R} \dot{\mathbf{q}}$ \\
\hline$P_{5}^{q}$ & 2 & body velocity ${ }_{B} \mathbf{v}$ & {$\left[\begin{array}{ll}\mathbf{S}_{x y B} \mathbf{J} & \mathbf{0}\end{array}\right]$} & $\mathbf{k}_{d}^{x y}\left({ }_{B} \mathbf{v}_{d e s}-{ }_{B} \mathbf{v}\right)-\mathbf{S}_{x y B} \dot{\mathbf{J}} \dot{\mathbf{q}}$ \\
\hline
\end{tabular}

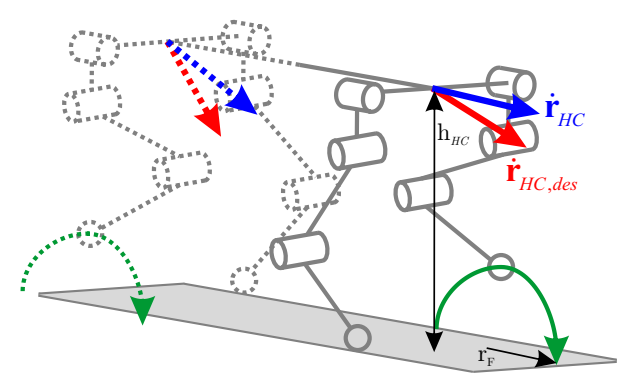

(a) Foot placement control

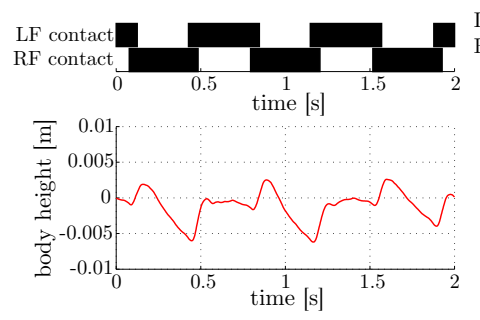

(b) Walking Trot

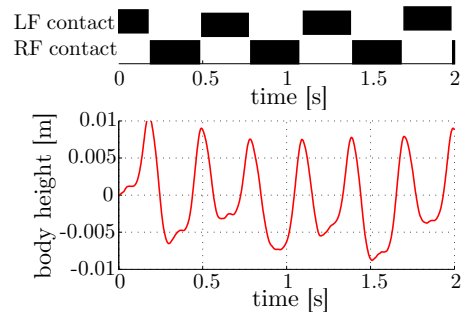

(c) Running Trot

Figure 9: Reactive foot placement (a) in combination with the whole body controller allows to stabilize walking (b) and running (c) trot. c) shows a short flight phase, a higher stride frequency, and larger oscillations in the body height.

\subsection{Dynamic Running}

This section outlines the application of the reduced control approach summarized in Table 2 to enable dynamic locomotion.

\subsubsection{Motion Generation}

Motion generation respectively foothold planning for dynamic gaits is based on (Coros et al., 2011). Starting from a gait graph that describes the timing for the footfalls (e.g. Figure 9(b)], we use a time-normalized phase $\omega \in[0,1]$ to define the swing leg position and body posture. The desired foothold locations are calculated in a similar process as introduced by Raibert (1986) and illustrated in Figure 9(a).

$$
\mathbf{r}_{F}=k_{p}^{F B}\left(\dot{\mathbf{r}}_{H C, \mathrm{des}}-\dot{\mathbf{r}}_{H C}\right) \sqrt{\frac{h_{H C}}{g}}+k_{p}^{F F}\left(\frac{1}{2} \dot{\mathbf{r}}_{H C, \mathrm{des}} \Delta t_{\text {stance }}\right),
$$

with the feedback gain $k_{p}^{F B}>0$, the desired velocity $\dot{\mathbf{r}}_{H C \text {,des }}$, the reference velocity at the front respectively rear hip location $\dot{\mathbf{r}}_{H C}$, as well as the feedforward gain $k_{p}^{F B}<0$ and the corresponding stance duration $\Delta t_{\text {stance. }}$. While walking, the main body orientation is kept constant and the base position is continuously moved forward with a superimposed vertical oscillation $z(\omega)$ to achieve a natural looking motion. For more details, the reader is referred to work done by Gehring et al. (2013).

\subsubsection{Running and Walking Trot (Exp10, Exp11)}

As an intuitive set of user or high level control input, the desired robot speed in sagittal ${ }_{B} \dot{x}\left({ }_{B} \mathbf{e}_{x}\right.$ forwards direction in main body frame) and coronal direction ${ }_{B} \dot{y}\left({ }_{B} \mathbf{e}_{y}\right.$ sidewards direction in main body frame), as well as the turning rate ${ }_{I} \dot{\psi}$ about a inertial frame fixed vertical direction ${ }_{I} \mathbf{e}_{z}$ are selected. According to these set values summarized in Table 7 the hierarchical task-space problem is described in different reference frames using the translation Jacobian ${ }_{I} \mathbf{J}=\frac{\partial_{I} \mathbf{r}}{\partial \mathbf{q}}$ and rotation Jacobian ${ }_{I} \mathbf{J}_{R}=\frac{\partial_{I} \boldsymbol{\Omega}}{\partial \dot{\mathrm{g}}}$ expressed in the inertial frame $I$ and the transformation matrix $\mathbf{C}_{B I}$ from the inertial frame $I$ to the body fixed frame $B$ such that ${ }_{B} \mathbf{J}=\mathbf{C}_{B I I} \mathbf{J} . \mathbf{S}_{i}$ express selection matrices with unit entries for the selected directions.

The application of this method in simulations as well in real world experiments required only minimal and intuitive gain tuning. The system was successfully tested in a walking trot (Exp10, Figure 9(b)) with a short contact transition phase as well as in a running trot (Exp11, Figure 9(c) that includes a full flight phase. With only the diagonal pairs of legs in simultaneous ground contact, StarlETH experiences truly-underactuated phases $\left(n_{u}=1\right)$ whereby the angular momentum around the line of support cannot be regulated. Hence, we choose the main body velocity as task with lowest priority. This means that the desired velocity is only tracked as well as possible (in a least square sense). However, as the robot can additionally compensate for velocity errors by the foot placement controller (62), the entire motion is still robustly stabilized. 


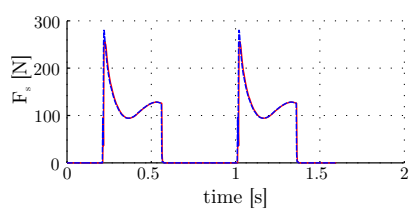

(a) LF vertical contact forces

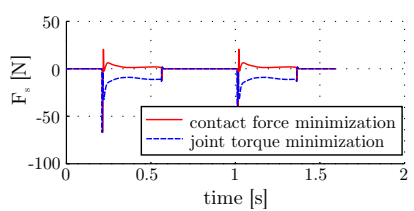

(b) LF horizontal contact forces

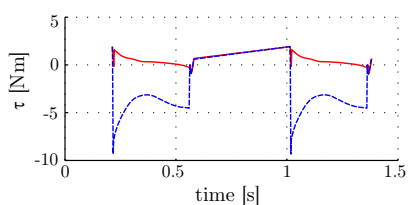

(c) LF HFE torque

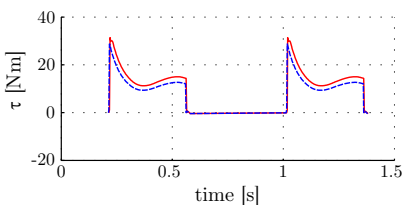

(d) LF KFE torque

Figure 10: Force and torque distribution with different optimization goals with $\mathbf{W}_{\tau}=\mathbf{I}$ (blue dotted line) or $\mathbf{W}_{F}=\mathbf{I}$ (red solid) as $P_{2}^{\tau}$. When optimizing for motor efficiency (blue dotted), the load of the knee is lowered and part is taken by the hip motor. In return the contact force increases in horizontal direction and hence also the risk of slippage.

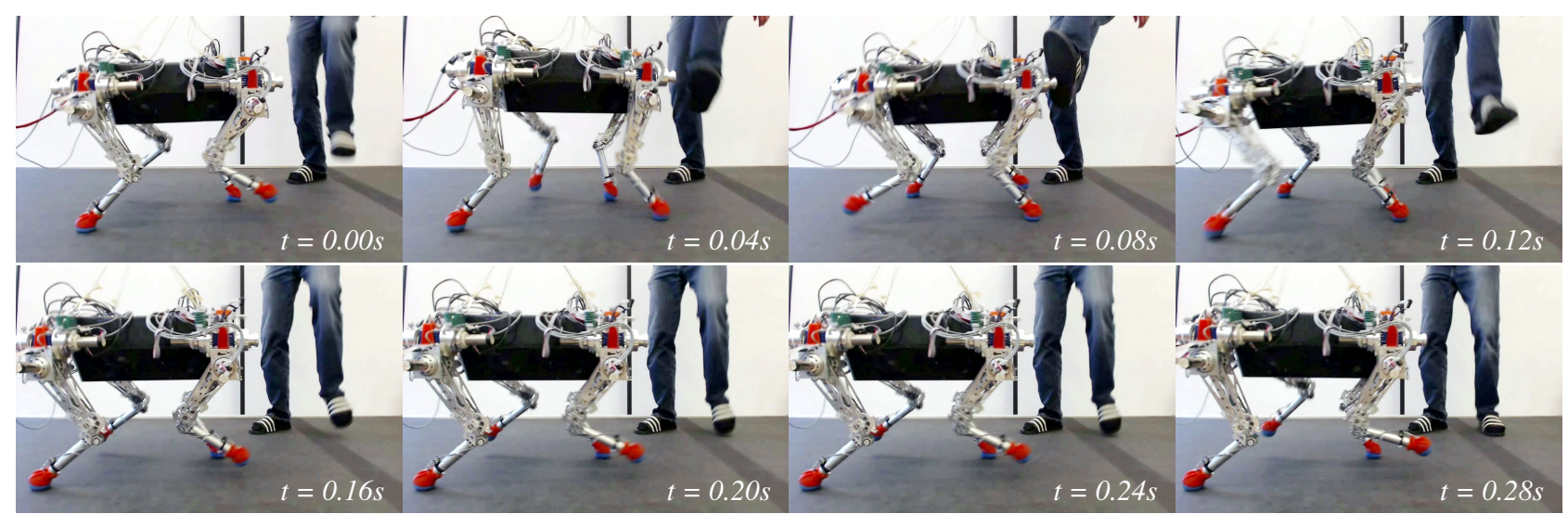

Figure 11: Exp12: StarlETH compensates for external disturbances such as a kick while trotting by stepping sideward and generating a net counterforce.

\subsubsection{Force and Torque Optimization (Sim11)}

Having only two feet in contact with the ground, the space for optimizing the contact forces or joint torques reduces to a single dimension. We tested both, minimizing joint torques $\left(\mathbf{W}_{\tau}=\mathbf{I}\right)$ as well as contact forces $\left(\mathbf{W}_{F}=\mathbf{I}\right)$. As indicated in Figure 10, the different optimization strategies slightly change the load distribution while showing the same motion. When minimizing the joint torque $\left(\mathbf{W}_{\tau}=\mathbf{I}\right.$, blue dashed), the mean friction coefficient for in-place trotting is $\bar{\mu}=0.14$, minimizing the contact force $\left(\mathbf{W}_{F}=\mathbf{I}\right.$, red solid) lowers this to $\bar{\mu}=0.05$ but in return increases the actuator cost 60 ) about $20 \%$.

\subsubsection{Stability and Robustness (Exp12)}

Stability and robustness usually determine how well a system can compensate for disturbances such as external pushes or unknown obstacles. In contrast to linear control theory that has well elaborated tools to measure this property, robustness is hard to quantify for legged systems. Maximal disturbances before tipping over can be analytically determined for static walking as the center of pressure is not allowed to coincide with the boundary of the support triangle. This changes when analyzing dynamic gaits, in our example trotting gaits with just the diagonal pair of legs in contact. There are so many parameters of influence that a robustness analysis in most cases boils down to a qualitative experimental evaluation to identify the stability limitations.

In general, a dynamically balancing legged robot has two means to reject disturbances. First, all legs in contact can produce reaction forces to counteract the perturbations as well as possible. Second, to compensate for disturbances in the underactuated subspace, the contact point locations are adapted accordingly. As an example depicted in Figure 11, we kicked the robot from the side. This causes a velocity error at the corresponding hip center in coronal direction and hence, due to 62, StarlETH steps sidewards to compensate. Thereby, it produces a counterforce and, within two steps, finds back to the nominal trotting gait.

\section{Conclusion}

This paper outlines a generalized framework for hierarchical task-space inverse dynamics control based on least squares optimization. Taking inspiration from existing work on task prioritization and inverse dynamics control, the behavior of a complex (legged) robot is described by a set of motion, torque, or contact force objectives with different priorities. This control problem can be solved by iterative null-space projection or numerical QP solvers. Moreover, projecting the system dynamics into the support or actuator null-space allows to reduce the optimization dimensionality. Instead 
of combined optimization of the linearly dependent generalized accelerations, actuator torques, and contact forces, the least squares problems can also be solved as a sequence of kinematic motion optimization and inverse dynamics with additional (prioritized) tasks to optimize the internal contact forces. This latter method has the advantage that it requires only inversion of kinematic system properties.

The main contribution of the present work is the experimental validation of the outlined whole-body controller. Given the precisely torque controllable quadrupedal robot StarlETH, this represents, to the best of our knowledge, the first hardware experiment of locomotion with pure OSC (i.e., no local joint position regulation). In an extensive study, we presented how contact point redundancy in static walking can be exploited to minimize energy consumption or to reduce the risk of slippage while walking in uneven terrain. We outlined an interpolation method that guarantees smooth contact transitions and include inequality tasks for joint angle and actuator torque limitations. The experimental section is further extended to dynamic trotting gaits, showing that the method works also for truly-underactuated systems. Hereby, a clear hierarchy in the motion part ensures that important directions like body orientation or vertical motion are executed by all means while translational motion remains less important and is regulated on a step-to-step basis by appropriate foothold placement. It is hence only tracked as good as possible in a least squares sense. The proposed control structure ensures stable running even under significant external disturbances by uneven ground or artificial pushes.

The presented results with pure task-space control and onboard state estimation constitute a strong argument that whole-body, respectively full inverse dynamics techniques, are very useful to enable sophisticated behaviors of a new generation of torque controlled (legged) robots.

\section{A Index to Multimedia Extensions}

The multimedia extensions to this article are at: http://www.ijrr.org.

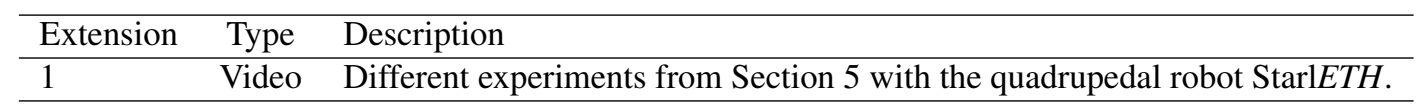

\section{References}

F. Aghili. A unified approach for inverse and direct dynamics of constrained multibody systems based on linear projection operator: applications to control and simulation. IEEE Transactions on Robotics, 21(5):834-849, 2005. URL http: //dx.doi.org/10.1109/TRO.2005.851380

A. k. Björck. Numerical Methods for Least Squares Problems. Society for Industrial and Applied Mathematics, Jan. 1996. ISBN 978-0-89871-360-2. doi: 10.1137/1.9781611971484. URL http://dx.doi.org/10.1137/1. 9781611971484 .

M. Bloesch, M. Hutter, M. H. Hoepflinger, S. Leutenegger, C. Gehring, C. D. Remy, and R. Siegwart. State Estimation for Legged Robots - Consistent Fusion of Leg Kinematics and IMU. In Robotics Science and Systems (RSS), Sydney, Australia, 2012. URL http://www. roboticsproceedings.org/rss08/p03.html.

J. Buchli, M. Kalakrishnan, M. Mistry, P. Pastor, and S. Schaal. Compliant quadruped locomotion over rough terrain. In IEEE/RSJ International Conference on Intelligent Robots and Systems (IROS), pages 814-820. IEEE, Oct. 2009. ISBN 978-1-4244-3803-7. doi: 10.1109/IROS.2009.5354681. URL http://ieeexplore.ieee.org/ lpdocs/epic03/wrapper.htm?arnumber $=5354681$.

S. Coros, A. Karpathy, B. Jones, L. Reveret, and M. van de Panne. Locomotion skills for simulated quadrupeds. In ACM SIGGRAPH, Vancouver, British Columbia, Canada, 2011. ACM. doi: 10.1145/1964921.1964954. URL http: //dx.doi.org/10.1145/1964921.1964954.

M. de Lasa and A. Hertzmann. Prioritized optimization for task-space control. IEEE/RSJ Intenational Conference on Intelligent Robots and Systems (IROS), pages 5755-5762, Oct. 2009. doi: 10.1109/IROS.2009.5354341. URL http: //ieeexplore.ieee.org/lpdocs/epic03/wrapper.htm?arnumber=5354341.

A. Dietrich, T. Wimbock, A. Albu-Schaffer, and G. Hirzinger. Integration of Reactive, Torque-Based Self-Collision Avoidance Into a Task Hierarchy. IEEE Transactions on Robotics, 28(6):1278-1293, 2012. doi: 10.1109/tro.2012. 2208667. URL http://dx.doi.org/10.1109/TRO.2012.2208667.

A. Escande, N. Mansard, and P.-B. Wieber. Fast resolution of hierarchized inverse kinematics with inequality constraints. In IEEE International Conference on Robotics and Automation (ICRA), pages 3733-3738. IEEE, May 2010. ISBN 978-1-4244-5038-1. doi: 10.1109/ROBOT.2010.5509953. URL http://ieeexplore.ieee.org/lpdocs/ epic03/wrapper.htm?arnumber $=5509953$. 
P. Fankhauser, M. Hutter, C. Gehring, and C. D. Remy. Optimizing Robotic Single Legged Locomotion with Reinforcement Learning. Master thesis, 2012. URL/http://students.asl.ethz.ch/upl_pdf/411-report.pdf.

R. Featherstone. An Empirical Study of the Joint Space Inertia Matrix. The International Journal of Robotics Research (IJRR), 23(9):859-871, 2004. doi: 10.1177/0278364904044869. URL http://dx.doi.org/10.1177/ 0278364904044869 .

C. Gehring, S. Coros, M. Hutter, M. Bloesch, M. H. Hoepflinger, and R. Siegwart. Control of Dynamic Gaits for a Quadrupedal Robot. In IEEE International Conference on Robotics and Automation (ICRA), Karlsruhe, Germany, 2013.

P. G. Gonzales de Santos, E. Garcia, and J. Estremera. Stability in Walking Robots. In Quadrupedal Locomotion. An Introduction to the Control of Four-legged Robots, volume 1, pages 33-54. Springer, Berlin, 2006.

H. Hanafusa, T. Yoshikawa, and Y. Nakamura. Analysis and control of articulated robot with redundancy. In IFAC Symposium on Robot Control, volume 4, pages 1927-1932, 1981.

A. Herzog, L. Righetti, F. Grimminger, P. Pastor, and S. Schaal. Momentum-based Balance Control for Torque-controlled Humanoids. URL http://arxiv.org/abs/1305.2042,

G. Hirzinger, A. Albu-Schaffer, M. Hahnle, I. Schaefer, and N. Sporer. On a new generation of torque controlled lightweight robots. In International Conference on Robotics and Automation (ICRA), volume 4, pages 3356-3363, 2001. doi: 10.1109/ROBOT.2001.933136. URL http://dx.doi.org/10.1109/ROBOT.2001.933136

M. Hutter. StarlETH \& Co - Design and Control of Legged Robots with Compliant Actuation. PhD thesis, ETH Zurich, 2013.

M. Hutter, C. Gehring, and R. Siegwart. proNEu: Derivation of Analytical Kinematics and Dynamics. Technical report, Autonomous Systems Lab, ETHZ, 2011a. URL http://www.leggedrobotics.ethz.ch/lib/exe/ fetch.php?media=research:proneu_v10_documentation.pdf.

M. Hutter, C. D. Remy, M. H. Hoepflinger, and R. Siegwart. High Compliant Series Elastic Actuation for the Robotic Leg ScarlETH. In International Conference on Climbing and Walking Robots (CLAWAR), pages 507-514, Paris, Fr, $2011 \mathrm{~b}$. doi: 10.1142/9789814374286\_0059. URL http://dx.doi.org/10.1142/9789814374286_0059.

M. Hutter, C. Gehring, M. Bloesch, M. H. Hoepflinger, C. D. Remy, and R. Siegwart. StarlETH: a Compliant Quadrupedal Robot for Fast, Efficient, and Versatile Locomotion. In International Conference on Climbing and Walking Robots (CLAWAR), pages 483-490, 2012a. doi: 10.1142/9789814415958\_0062. URL http://dx.doi.org/10.1142/ 9789814415958_0062.

M. Hutter, M. H. Hoepflinger, C. D. Remy, and R. Siegwart. Hybrid Operational Space Control for Compliant Legged Systems. In RSS Robotics Science and Systems, Sydney, Australia, 2012b. URL http: // roboticsproceedings . org/rss08/p17.pdf.

O. Kanoun, F. Lamiraux, and P.-B. Wieber. Kinematic Control of Redundant Manipulators: Generalizing the TaskPriority Framework to Inequality Task. IEEE Transactions on Robotics, 27(4):785-792, Aug. 2011. ISSN $1552-3098$. doi: 10.1109/TRO.2011.2142450. URL http://ieeexplore.ieee.org/lpdocs/epic03/wrapper. htm? arnumber $=5766760$.

O. Khatib. A unified approach for motion and force control of robot manipulators: The operational space formulation. IEEE Journal of Robotics and Automation, 3(1):43-53, 1987. URL http: / / dx . doi . org/10.1109/JRA. 1987.1087068 .

N. Mansard. A dedicated solver for fast operational-space inverse dynamics. In IEEE International Conference on Robotics and Automation (ICRA), pages 4943-4949. IEEE, May 2012. ISBN 978-1-4673-1405-3. doi: 10.1109/ICRA.2012.6224851. URL http://ieeexplore.ieee.org/lpdocs/epic03/wrapper.htm? arnumber $=6224851$.

N. Mansard, O. Khatib, and A. Kheddar. A Unified Approach to Integrate Unilateral Constraints in the Stack of Tasks. IEEE Transactions on Robotics, 25(3):670-685, June 2009. ISSN 1552-3098. doi: 10.1109/TRO.2009.2020345. URL http://ieeexplore.ieee.org/lpdocs/epic03/wrapper.htm?arnumber=4926146.

R. B. McGhee, A. A. Fkask, and A. Frank. On the stability properties of quadruped creeping gaits. Mathematical Biosciences, 3:331-351, Aug. 1968. ISSN 00255564. doi: 10.1016/0025-5564(68)90090-4. URL http://dx . doi.org/10.1016/0025-5564(68)90090-4. 
M. Mistry, J. Buchli, and S. Schaal. Inverse Dynamics Control of Floating Base Systems Using Orthogonal Decomposition. In International Conference on Robotics and Automation (ICRA), pages 3406-3412, 2010. ISBN 1050-4729. URL http://dx.doi.org/10.1109/ROBOT.2010.5509646.

Y. Nakamura, H. Hanafusa, and T. Yoshikawa. Task-Priority Based Redundancy Control of Robot Manipulators. The International Journal of Robotics Research, 6(2):3-15, June 1987. ISSN 0278-3649. doi: 10.1177/ 027836498700600201. URL http://dx.doi.org/10.1177/027836498700600201.

J. Nakanishi, R. Cory, M. Mistry, J. Peters, and S. Schaal. Operational Space Control: A Theoretical and Empirical Comparison. The International Journal of Robotics Research, 27(6):737-757, June 2008. ISSN 0278-3649. doi: 10.1177/0278364908091463. URL http://dx.doi.orgi/10.1177/0278364908091463

R. Philippsen, L. Sentis, O. Khatib, and L. Sentist. An open source extensible software package to create whole-body compliant skills in personal mobile manipulators. In IEEE/RSJ International Conference on Intelligent Robots and Systems (IROS), pages 1036-1041, 2011. ISBN 2153-0858. URL http://dx.doi.org/10.1109/IROS.2011. 6095163 .

M. H. Raibert. Legged robots that balance. MIT Press, Cambridge, Mass., 1986. ISBN 0262181177.

O. E. Ramos, N. Mansard, O. Stasse, and P. Souères. Walking on Non-planar Surfaces using an Inverse Dynamic Stack of Tasks. In IEEE-RAS International Conference on Humanoid Robots, 2012.

C. D. Remy, K. W. Buffinton, and R. Siegwart. Comparison of Cost Functions for Electrically Driven Running Robots. In IEEE International Conference on Robotics and Automation (ICRA), pages 2343-2350. IEEE, May 2012. ISBN 978-1-4673-1405-3. doi: 10.1109/ICRA.2012.6224960. URL http://dx.doi.org/10.1109/ICRA.2012. 6224960 .

L. Righetti, J. Buchli, M. Mistry, and S. Schaal. Inverse dynamics with optimal distribution of ground reaction forces for legged robots. In international conference on climbing and walking robots (CLAWAR), 2010.

L. Righetti, J. Buchli, M. Mistry, and S. Schaal. Inverse dynamics control of floating-base robots with external constraints: A unified view. In International Conference on Robotics and Automation (ICRA), pages 1085-1090, 2011a. ISBN 1050-4729. URL http://dx.doi.org/10.1109/ICRA.2011.5980156.

L. Righetti, J. Buchli, M. Mistry, and S. Schaal. Control of legged robots with optimal distribution of contact forces. In IEEE-RAS International Conference on Humanoid Robots (Humanoids), pages 318-324, 2011b. ISBN 2164-0572. doi: 10.1109/Humanoids.2011.6100832. URL http://dx.doi.org/10.1109/Humanoids.2011.6100832.

L. Righetti, J. Buchli, M. Mistry, M. Kalakrishnan, and S. Schaal. Optimal distribution of contact forces with inverse dynamics control. International Journal of Robotics Research (IJRR), 2013. doi: 10.1177/0278364912469821. URL http://ijr.sagepub.com/content/early/2013/01/22/0278364912469821.abstract.

L. Saab, N. Mansard, F. Keith, J.-Y. Fourquet, and P. Soueres. Generation of dynamic motion for anthropomorphic systems under prioritized equality and inequality constraints. In IEEE International Conference on Robotics and Automation (ICRA), pages 1091-1096. IEEE, May 2011a. ISBN 978-1-61284-386-5. doi: 10.1109/ICRA.2011.5980384. URL http://ieeexplore.ieee.org/lpdocs/epic03/wrapper.htm?arnumber=5980384

L. Saab, O. Ramos, N. Mansard, P. Soueres, and J.-Y. Fourquet. Generic dynamic motion generation with multiple unilateral constraints. In IEEE/RSJ International Conference on Intelligent Robots and Systems (IROS), pages 4127-4133. IEEE, Sept. 2011b. ISBN 978-1-61284-456-5. doi: 10.1109/IROS.2011.6094795. URL http: //ieeexplore.ieee.org/lpdocs/epic03/wrapper.htm?arnumber=6094795.

L. Saab, O. E. Ramos, F. Keith, N. Mansard, P. Soueres, and J.-Y. Fourquet. Dynamic Whole-Body Motion Generation Under Rigid Contacts and Other Unilateral Constraints. IEEE Transactions on Robotics, 29(2):346-362, Apr. 2013. ISSN 1552-3098. doi: 10.1109/TRO.2012.2234351. URL/http://ieeexplore.ieee.org/lpdocs/ epic03/wrapper.htm?arnumber $=6482266$

E. Saltzman and J. A. S. Kelso. Skilled Actions: A Task Dynamic Approach. Psychological Review, 94(1):84-106, 1987.

C. Samson, M. L. Borgne, and B. Espiau. Robot Control: The Task Function Approach. Clarendon Press, Oxford, U.K., 1991.

S. Schaal. The SL Simulation and Real-Time Control Software Package. Technical report, 2009.

S. Schaal and N. Schweighofer. Computational motor control in humans and robots. Current Opinion in Neurobiology, 15(6):675-682, 2005. doi: DOI10.1016/j.conb.2005.10.009. URL <GotoIS I > / / 000234167700009 http: //dx.doi.org/10.1016/j.conb.2005.10.009. 
J. P. Scholz and G. Schoner. The uncontrolled manifold concept: identifying control variables for a functional task. Experimental Brain Research, 126(3):289-306, 1999. URL/http://www.ncbi.nlm.nih.gov/pubmed/ 10382616http://dx.doi.org/10.1007/s002210050738.

L. Sentis. Synthesis and Control of Whole-Body Behaviors in Humanoid Systems. PhD thesis, 2007.

L. Sentis and O. Khatib. Control of Free-Floating Humanoid Robots Through Task Prioritization. In IEEE International Conference on Robotics and Automation (ICRA), pages 1718-1723, 2005. doi: 10.1109/ROBOT.2005.1570361. URL http://dx.doi.org/10.1109/ROBOT.2005.1570361.

L. Sentis and O. Khatib. Compliant Control of Multicontact and Center-of-Mass Behaviors in Humanoid Robots. IEEE Transactions on Robotics, 26(3):483-501, June 2010. ISSN 1552-3098. doi: 10.1109/TRO.2010.2043757. URL http://ieeexplore.ieee.org/lpdocs/epic03/wrapper.htm?arnumber=5451086

B. Siciliano and J. J. E. Slotine. A General Framework for Managing Multiple Tasks in Highly Redundant Robotic Systems. In International Conference on Robots in Unstructured Environments (ICAR), pages 1211-1216 vol.2, 1991. doi: 10.1109/icar.1991.240390. URL http://dx.doi.org/10.1109/icar.1991.240390.

D. Zhou, K. H. Low, and T. Zielinska. An efficient foot-force distribution algorithm for quadruped walking robots. Robotica, 18(04):403-413, 2000. URL http://dx.doi.org/10.1017/S0263574799001824. 